\title{
Integrated genomic, epidemiologic investigation of Candida auris skin colonization in a skilled nursing facility
}

\author{
Diana M. Proctor ${ }^{1}{ }^{1}$, Thelma Dangana², D. Joseph Sexton ${ }^{3}$, Christine Fukuda², Rachel D. Yelin², \\ Mary Stanley², Pamela B. Bell2, Sangeetha Baskaran², Clay Deming1, Qiong Chen', Sean Conlan', \\ Morgan Park ${ }^{4}$, NISC Comparative Sequencing Program ${ }^{\star}$ Rory M. Welsh ${ }^{3}$, Snigdha Vallabhaneni ${ }^{3,5}$, \\ Tom Chiller ${ }^{3}$, Kaitlin Forsberg ${ }^{3}$, Stephanie R. Black ${ }^{6}$, Massimo Pacilli6, Heidi H. Kong ${ }^{7}$, \\ Michael Y. Lin², Michael E. Schoeny ${ }^{8}$, Anastasia P. Litvintseva ${ }^{3}$, Julia A. Segre ${ }^{1,9 凶}$ and \\ Mary K. Hayden $\mathbb{D}^{2,9 凶}$
}

Candida auris is a fungal pathogen of high concern due to its ability to cause healthcare-associated infections and outbreaks, its resistance to antimicrobials and disinfectants and its persistence on human skin and in the inanimate environment. To inform surveillance and future mitigation strategies, we defined the extent of skin colonization and explored the microbiome associated with $C$. auris colonization. We collected swab specimens and clinical data at three times points between January and April 2019 from 57 residents (up to ten body sites each) of a ventilator-capable skilled nursing facility with endemic C. auris and routine chlorhexidine gluconate (CHG) bathing. Integrating microbial-genomic and epidemiologic data revealed occult $\mathrm{C}$. auris colonization of multiple body sites not targeted commonly for screening. High concentrations of CHG were associated with suppression of $\boldsymbol{C}$. auris growth but not with deleterious perturbation of commensal microbes. Modeling human mycobiome dynamics provided insight into underlying alterations to the skin fungal community as a possible modifiable risk factor for acquisition and persistence of $C$. auris. Failure to detect the extensive, disparate niches of $C$. auris colonization may reduce the effectiveness of infection-prevention measures that target colonized residents, highlighting the importance of universal strategies to reduce $C$. auris transmission.

C auris has emerged as a human pathogen of high concern due to its extensive antifungal resistance, high mortality rates associated with invasive infections and its potential to cause healthcare-associated outbreaks ${ }^{1-3}$. A recent systematic review of nearly 5,000 cases of C. auris from 33 countries reported an overall crude mortality rate of $39 \%^{4}$. The US Centers for Disease Control and Prevention (CDC) have declared C. auris to be an urgent public health threat, the first fungal pathogen to receive this designation $^{5}$. Persistent skin colonization ${ }^{6}$ likely contributes to the epidemic potential of C. auris. Healthcare providers may contaminate their hands, gloves or clothing with C. auris by touching the skin of a colonized patient. C. auris can then be transmitted to another patient if the healthcare provider fails to perform adequate hand hygiene or adhere to other infection-prevention measures. Additionally, shedding from colonized patients' skin into the healthcare environment, calculated to occur at a rate of a million microbes per hour ${ }^{7}$, creates an environmental reservoir and source of ongoing nosocomial transmission $^{8-11}$. In the US, high-acuity long-term care facilities such as long-term acute care hospitals and ventilator-capable skilled nursing facilities have reported high prevalence of C. auris, and there is concern that these facilities may serve as drivers of regional C. auris spread ${ }^{2,3,12,13}$.

Effective infection-control measures play a central role in the management of $C$. auris because frequent resistance to multiple classes of antifungal agents (that is, azoles, polyenes, echinocandins) limits treatment options for invasive infection ${ }^{14-16}$. Constraining effective infection control is the paucity of published data concerning the spatial extent of skin colonization by $C$. auris and clarity on the relative sensitivity of different body sites to identify residents colonized with $C$. auris. Occult colonization has been shown to underlie transmission in recalcitrant outbreaks involving other pathogens, including Klebsiella pneumoniae ${ }^{17}$. Efficacy of the bisbiguanide antiseptic CHG in controlling C. auris colonization is another uncertainty. While CHG bathing is a common component of multimodal C. auris control programs in acute and long-term care settings, previous investigators have reported persistent colonization of residents, environmental contamination and ongoing C. auris transmission despite CHG bathing ${ }^{9,12,18}$. 
Table 1 | Bivariate associations of clinical characteristics with C. auris-colonization status at the time of the first survey

\begin{tabular}{|c|c|c|c|c|}
\hline & Colonized $(n=46)$ & Not colonized $(n=11)$ & OR $(95 \% \mathrm{Cl})$ & $P$ value \\
\hline $\begin{array}{l}\text { Length of stay }(d) \text { between admission and first } \\
\text { swab }(M \pm I Q R)\end{array}$ & $361 \pm 801$ & $571 \pm 1,372$ & $1.00(0.99,1.00)$ & 0.26 \\
\hline Age in years (mean \pm s.d.) & $59 \pm 14$ & $59 \pm 14$ & $1.00(0.95,1.05)$ & 0.89 \\
\hline Female $(n(\%))$ & $18(39)$ & $5(45)$ & $0.77(0.20,2.91)$ & 0.74 \\
\hline African American ( $n(\%))$ & $31(74)$ & $9(90)$ & $0.31(0.066,2.76)$ & 0.42 \\
\hline Mechanical ventilation $(n(\%))$ & $23(50)$ & $0(0)$ & $23.00(1.28,413.3)$ & 0.002 \\
\hline Tracheostomy (n (\%)) & $34(74)$ & $6(55)$ & $2.36(0.61,9.17)$ & 0.27 \\
\hline Urinary catheter $(n(\%))$ & $33(72)$ & $3(27)$ & $6.77(1.55,29.56)$ & 0.012 \\
\hline Hospital stay within past $90 \mathrm{~d}(n(\%))$ & $20(44)$ & $3(27)$ & $2.13(0.50,9.11)$ & 0.5 \\
\hline Charlson comorbidity index $(M \pm I Q R)$ & $3 \pm 2$ & $3 \pm 2$ & $0.91(0.65,1.28)$ & 0.59 \\
\hline Congestive heart failure $(n(\%))$ & $10(22)$ & $5(45)$ & $0.33(0.084,1.32)$ & 0.11 \\
\hline Antibacterial receipt in prior $90 \mathrm{~d}(n(\%))$ & $34(74)$ & $5(45)$ & $3.4(0.88,13.21)$ & 0.030 \\
\hline Antifungal receipt in prior $90 \mathrm{~d}(n(\%))$ & $5(11)$ & $0(0)$ & $3.05(0.16,59.29)$ & 0.57 \\
\hline
\end{tabular}

aThe Braden Scale for Predicting Pressure Sore Risk assesses a person's risk of developing a pressure ulcer by examining six criteria: moisture, activity, mobility, nutrition and friction and shear. bScreening of facility residents for carbapenemase-producing bacterial colonization was performed routinely. Screening for colonization by other multidrug-resistant organisms was not performed. $\mathrm{Cl}$, confidence interval; $\mathrm{IQR}$, interquartile range; $\mathrm{M}$, median; $\mathrm{OR}$, odds ratio.

C. auris was first detected in metropolitan Chicago, IL, in 2016, where post-acute care facilities have become epicenters for this pathogen ${ }^{3,12}$. Here we report on an integrated clinical research program to study $C$. auris colonization of residents at a ventilator-capable skilled nursing facility in Chicago with endemic C. auris and routine $\mathrm{CHG}$ bathing. We conducted serial point prevalence surveys of skin, oral and perianal sites to investigate C. auris using culture-dependent and culture-independent methods and analyzed the relationship of CHG concentration with growth of $C$. auris and the skin microbiota. Phylogenetic marker genes of bacterial and fungal communities (16S rRNA and internal transcribed spacer (ITS)1, respectively) permit high-resolution investigation into the microbiome associated with clinical samples. To understand community dynamics of $C$. auris skin colonization, we investigated the temporal stability and diversity of skin, oral and gut bacterial and fungal communities.

\section{Results}

Overview of study participants. We conducted three monthly point prevalence surveys between January and April 2019 on the ventilator ward of a skilled nursing facility in Chicago, IL. At the time of each survey, research personnel collected swab specimens from up to ten body sites (anterior nares, external auditory canal, axilla, inguinal crease, perianal skin, toe web, palm and fingertips, buccal mucosa and tongue and tracheostomy site) for semiquantitative $(n=1,358)$ and quantitative $(n=424)$ C. auris culture, microbiome analysis $(n=1,358)$ and quantification of CHG concentration $(n=813)$ (Supplementary Table 1). Clinical data were collected at bedside from electronic medical records and recorded onto standardized data-collection forms. Fifty-seven (72\%) of 79 unique residents on the ward during the study period participated, and, of those participating, 49 (86\%) residents were found to be colonized with C. auris at one or more body site in one or more survey. Overall, residents were older adults, had a history of colonization or infection with carbapenem-resistant bacteria and had long lengths of stay before the first date of sample collection (Table 1). Residents with $C$. auris colonization were more likely to require an invasive medical device (for example, gastrostomy tube, mechanical ventilation) and were more likely to have received antibacterial agents in the $90 \mathrm{~d}$ before the first sample collection. While no participant developed C. auris clinical infection during the study period, review of clinical microbiology data from the time of admission through 6 months after the last survey identified one resident with $a C$. auris bloodstream infection 6 weeks before sample collection and two residents with C. auris bloodstream infections in the 6 months after the surveys concluded.

All isolates were susceptible to the commonly used antifungal agents fluconazole, amphotericin B and micafungin (Supplementary Table 2). Genomes and ITS1 sequences of $C$. auris isolates matched those of the South American clade IV ${ }^{19}$. Between isolates, the number of single-nucleotide variants ranged from one to 20 (mean $=14$, $N=4$ ) when reads were mapped to a nanopore reference genome generated from an isolate from this outbreak, consistent with clonality within the facility.

Patterns of body-site colonization with $C$. auris. To identify the spatial extent of $C$. auris colonization across the human body, we surveyed ten body sites with both culturing and microbial sequencing (Extended Data Fig. 1). Patterns of body-site colonization were highly individualized (Extended Data Fig. 2). Across all study participants, the frequency of colonization varied by body site: $42.9 \%$ of residents tested positive at nares, $40.4 \%$ of residents tested positive at palms and/or fingertips, and $35.7 \%$ of residents tested positive at toe webs (Fig. 1a). Importantly, residents who were culture negative at commonly targeted screening sites (here only unilaterally sampled axilla, inguinal crease and/or nares) were frequently colonized at other body sites, such as palms and/or fingertips. Of those who were colonized, $30.4 \%$ were colonized at a single body site, with monocolonization most commonly occurring at either the anterior nares or palms and/or fingertips (Extended Data Fig. 2). C. auris 
never monocolonized the buccal mucosa, tongue, neck, tracheostomy site or external auditory canal.

Because most colonized residents (69.6\%) were colonized at two or more sites, we next performed a sensitivity analysis to determine the combination of screening sites most effective at detecting the largest number of C. auris-colonized residents (Fig. 1b). Nares were the most sensitive single site, achieving $53.1 \%$ sensitivity. The most sensitive two-site combination was nares with palm and fingertips, with $76.1 \%$ sensitivity; adding toe webs increased sensitivity to $89.1 \%$, and adding the perianal site and the inguinal crease increased sensitivity to $97.8 \%$. Identification of all colonized residents was achieved by screening a minimum of six body sites (nares, palm and fingertips, toe webs, perianal skin, inguinal crease, axilla), consistent with the wide degree of variation observed in site-specific colonization with $C$. auris. The current recommended screening scheme collects a composite swab of a resident's bilateral axillae and inguinal creases ${ }^{20}$, sometimes with the addition of anterior nares ${ }^{21}$. In our study, which was performed with unilateral sampling, these two- and three-site combinations achieved sensitivities of $61.9 \%$ and $79.7 \%$, respectively.

As fungal load may contribute to a resident's risk of subsequent infection or of acting as a source of transmission through contaminating the environment or a healthcare provider, we quantified the bioburden of sites screened. The most densely colonized site was the nares, which exhibited a bimodal distribution with a median peak abundance of $10^{8.2}$ colony-forming units (CFU) (Extended Data Fig. 3). The axilla and inguinal crease harbored relatively low population densities: the axilla exhibited a uniform distribution with a median abundance of $10^{3.9} \mathrm{CFU}$, while the inguinal crease exhibited a bimodal distribution with two comparable peaks and a median abundance of $10^{4.9} \mathrm{CFU}$. Due to the semiquantitative nature of plate culture counts, quantitative analyses were performed. The median most probable number (MPN) of $C$. auris in nares was significantly higher than that in the axilla or inguinal crease, and nares had the greatest proportion of samples with a high bioburden (Fig. 1c and Extended Data Fig. 4). Collectively, these data suggest that multifocal colonization occurs commonly and that sites other than those screened most commonly may harbor high population densities, which may contribute to recalcitrant outbreaks experienced by some facilities.

Effectiveness of $\mathrm{CHG}$ against $\boldsymbol{C}$. auris colonization. The skin antiseptic CHG is used routinely for bathing hospital residents to reduce multidrug-resistant-organism colonization and infection.

Fig. 1 | Screening of multiple skin, nares, perianal and oral body sites for prevalence and individual-level bioburden of $\boldsymbol{C}$. auris colonization. $\mathbf{a}$, The proportion of residents colonized at each body site. $n=542$ independent samples of each of ten body sites of each of 57 residents at the time of the first screening. Data are presented as a point estimate $\pm 95 \%$ confidence intervals. An, perianal skin; Ax, axilla; Bu, buccal mucosa; Ea, external auditory canal; $\mathrm{Fg}$, palm and/or fingertips; Ic, inguinal crease; $\mathrm{N}$, anterior nares; Ne, neck; Tc, tracheostomy; To, tongue; Tw, toe web. b, Sensitivity analysis to calculate the proportion of colonized residents captured by screening different groupings of sites. Sensitivity is defined as the proportion colonized at each site grouping divided by the total number of residents identified as colonized at any body site. Dashed vertical lines correspond to the sensitivity of two routine screening strategies targeting the body sites axilla and inguinal crease (left dashed line) and axilla, inguinal crease and nares (right dashed line). A minimum of six sites was required to achieve $100 \%$ sensitivity, capturing all colonized individuals. c, Number of viable C. auris, determined by the MPN, plotted for the inguinal crease $(n=16)$, nares $(n=19)$ and axilla $(n=16)$. Groupwise medians are demarcated with black lines. Statistical significance of differences between sites was assessed with the Kruskal-Wallis test $(P<0.05)$.
Reports on the efficacy of CHG against colonization with C. auris have had mixed results ${ }^{9,22,23}$. As all residents of the ventilator-capable skilled nursing facility in the current study were undergoing routine

a

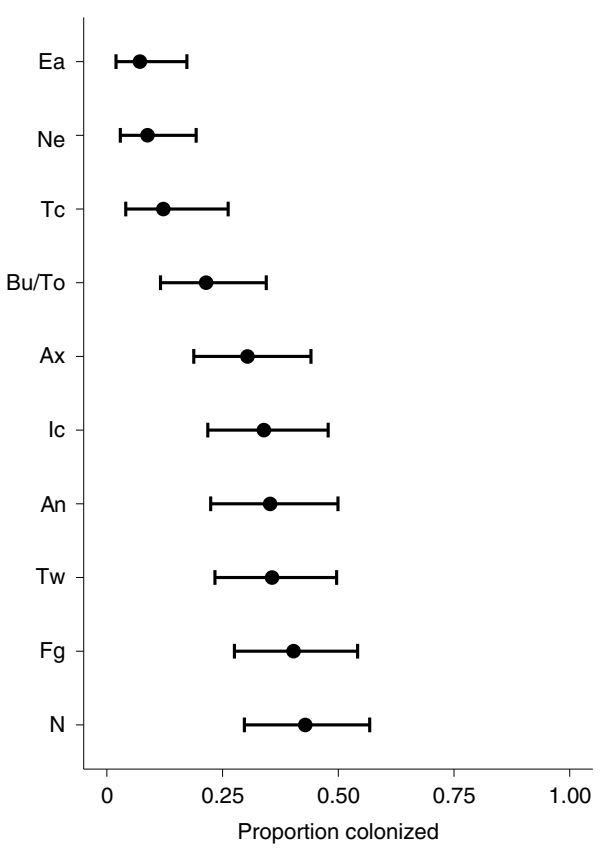

b

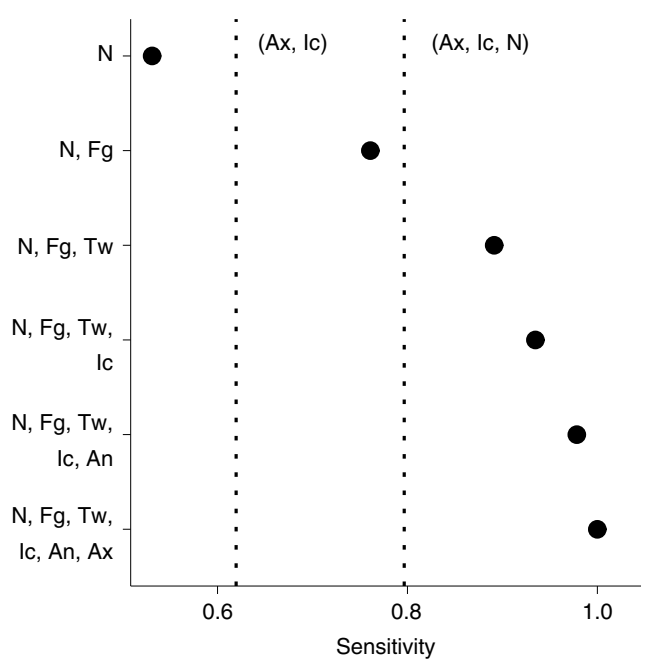

c

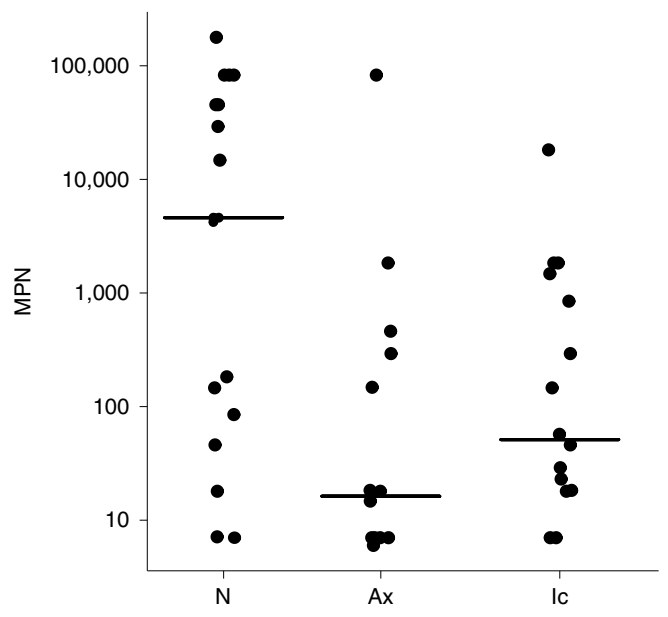



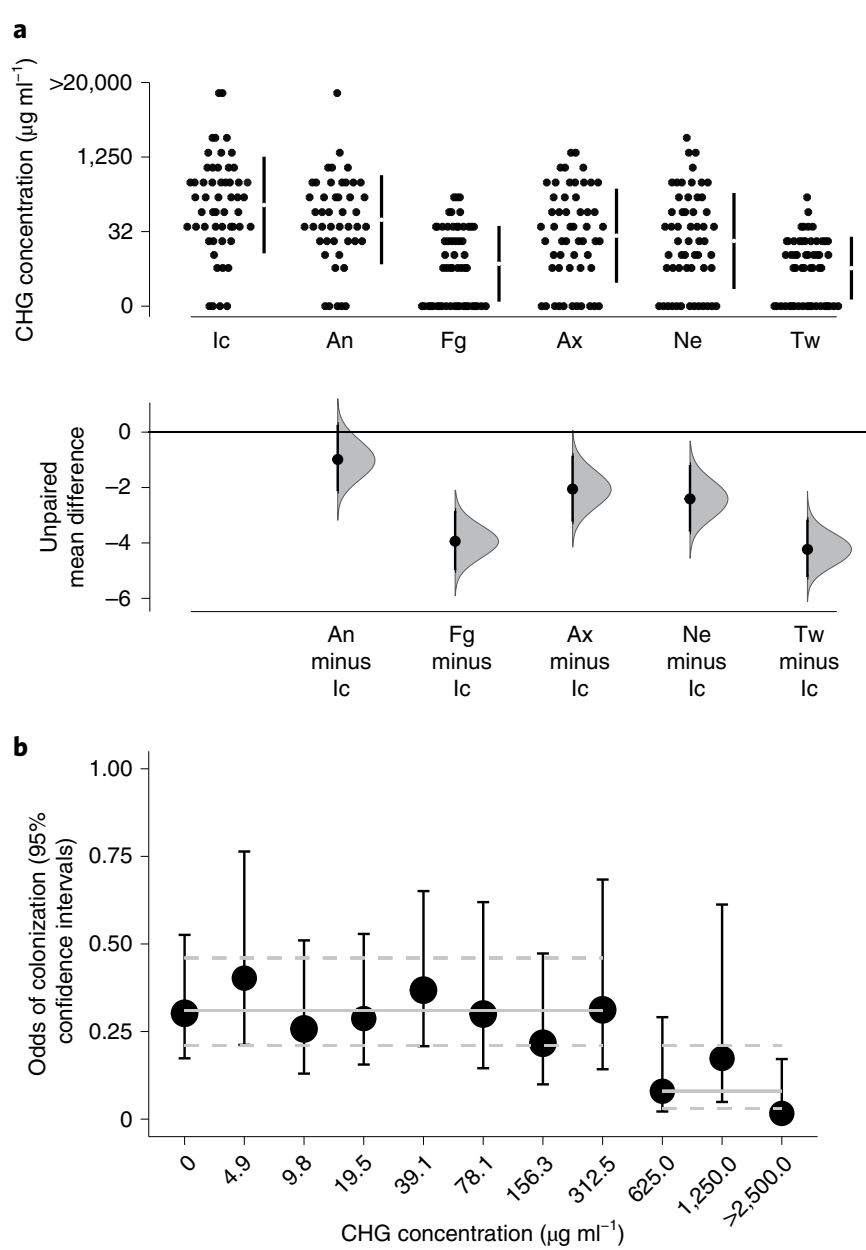

Fig. 2 | High concentrations of CHG are needed to reduce the odds of C. auris colonization. $\mathbf{a}$, Gardner-Altman estimation plot comparing the mean difference in $\mathrm{CHG}$ concentrations ( $\mu \mathrm{g} \mathrm{ml}^{-1}$ ) across body sites. Top, raw data are shown as a scatterplot of CHG concentration plotted as a function of body site for the first survey (54 residents, $n=319$ ). Bottom, data are presented as the mean difference between $\mathrm{CHG}$ concentration $\left(\mu \mathrm{g} \mathrm{ml}^{-1}\right)$ at each body site and the inguinal crease, the site reaching the highest average $\mathrm{CHG}$ concentration, $\pm 95 \%$ confidence intervals. Histograms reflect the sampling distribution from a nonparametric bootstrap. b. Each point represents the modeled odds of $C$. auris colonization ( $\pm 95 \%$ confidence intervals) plotted against the measured skin $\mathrm{CHG}$ concentration $\left(\mu \mathrm{g} \mathrm{ml}^{-1}\right)$, adjusted for multiple measurements within resident and over time. The solid horizontal lines represent odds of colonization per respective group (that is, $<625 \mu \mathrm{g} \mathrm{ml}^{-1}$ versus $\geq 625 \mu \mathrm{g} \mathrm{ml}^{-1}$ ), while the dashed lines encompass the $95 \%$ confidence interval surrounding each group estimate. Significance tests were based on the model-estimated log odds divided by the standard error, distributed as a t distribution.

bathing with 2\% CHG-impregnated cloths, we sought to investigate the relation between $\mathrm{CHG}$ concentration on skin and growth of $C$ auris in culture. Consistent with prior studies ${ }^{24}$, we found that CHG concentrations varied by body site $(F(5,740)=25.99, P<0.001$; Fig. 2a). The highest concentrations of CHG were measured at the inguinal crease (median and IQR, 78.1 and 39.1-312.5 $\mu \mathrm{g} \mathrm{ml}^{-1}$ ), while relatively low concentrations were measured at the palm and/ or fingertips (median and IQR, 9.8 and $0-39.1 \mu \mathrm{g} \mathrm{ml}^{-1}$ ) and the toe web (median and IQR, 4.9 and $0-19.5 \mu \mathrm{g} \mathrm{ml}^{-1}$ ). As we observed that sites with the lowest CHG concentrations (palm and/or fingertips, toe web) were colonized by $C$. auris at the highest frequencies
(Fig. 1), we tested for an explicit association. We found evidence of a modest but not significant linear association between $\mathrm{CHG}$ concentration ( $\log _{2}$ transformed) and the odds of $C$. auris detection (odds ratio, 0.93 ; 95\% confidence interval, $0.85-1.01 ; P=.068$ ). Cut-point analysis revealed a significant reduction in the odds of C. auris colonization for CHG concentrations $\geq 625 \mu \mathrm{g} \mathrm{ml}^{-1}$ (odds ratio, 0.27 ; 95\% confidence interval, $0.12-0.65 ; P=0.003$ ) (Fig. $2 b$ ). Importantly, the concentration of CHG needed to reduce the odds of colonization $\left(\geq 625 \mu \mathrm{g} \mathrm{ml}^{-1}\right)$ was detected at only $7.3 \%$ of skin sites tested. Because data on the time of the most recent bath before CHG measurement were not available, we could not control for this variable. Nonetheless, we note that the concentration of $625 \mu \mathrm{g} \mathrm{ml}^{-1}$, associated with reduced odds of $C$. auris colonization in situ, was 20-39 times higher than the minimum concentration of $\mathrm{CHG}$ required to inhibit the growth of $C$. auris in vitro $\left(16-32 \mu \mathrm{g} \mathrm{ml}^{-1}\right)$ (Supplementary Table 2).

Underlying microbiome associated with $C$. auris colonization. As $C$. auris must invade the microbiome to colonize human skin, we next explored associations between $C$. auris and features of the human microbiome. We characterized bacterial and fungal community composition by sequencing $16 \mathrm{~S}$ rRNA and ITS1 regions from clinical samples collected during the first survey at each of ten body sites of 51 residents. While foundational studies of young healthy volunteers demonstrated Malassezia predominance of the skin mycobiome ${ }^{25}$, the mycobiome of these skilled nursing facility residents was composed of multiple Malassezia and Candida species (Fig. 3a). Candida species found on the skin included a diverse constellation of C. auris, Candida tropicalis, Candida parapsilosis, Candida albicans, Candida orthopsilosis, Candida duobushaemulonii, Candida glabrata, Candida krusei, Candida dubliniensis and Candida metapsilosis, among others. Sites exhibiting potential for Candida predominance included perianal skin, the axilla, nares, the inguinal crease, palms and/or fingertips and toe webs, overlapping with sites identified as commonly colonized with $C$. auris by culturing. More extensive comparisons between culture and sequence results highlighted confounding underlying biological principles. For example, while culturing may demonstrate a higher C. auris level in the nares than in the inguinal crease (Fig. 1c), sequencing results may not identify $C$. auris as the predominant species due to the diversity, composition and bioburden of the nares microbiome (Fig. 3a).

Next, we sought to identify bacterial species enriched in C. auris-colonized samples. In a sparse, partial-least-squares discriminant analysis (sPLS-DA), Proteobacteria Proteus mirabilis, K. pneumoniae, Providencia stuartii and Pseudomonas aeruginosa contributed most to segregation of $C$. auris culture-positive samples away from C. auris culture-negative samples (Fig. 3b-d). Conversely, the skin commensal Staphylococcus hominis primarily contributed to the segregation of culture-negative samples away from culture-positive samples. Other commensal species, including Corynebacterium tuberculostearicum, Staphylococcus epidermidis, Staphylococcus caprae and Corynebacterium striatum, were also found to have principal-component loading scores associated with $C$. auris culture negativity. Notably, $P$. mirabilis and $P$. stuartii were among a small set of taxa that exhibited a direct correlation with CHG concentration, indicating an association between increasing relative abundance of these species and increasing CHG concentrations (Extended Data Fig. 5).

Longitudinal analysis of $C$. auris colonization. This longitudinal, observational clinical study was designed to explore microbiome alterations associated with $C$. auris acquisition and persistence. To explore mycobiome dynamics, the fungal community of samples from the monthly surveys was visualized by principal-coordinate analysis of the weighted UniFrac distance metric, which takes into 
a
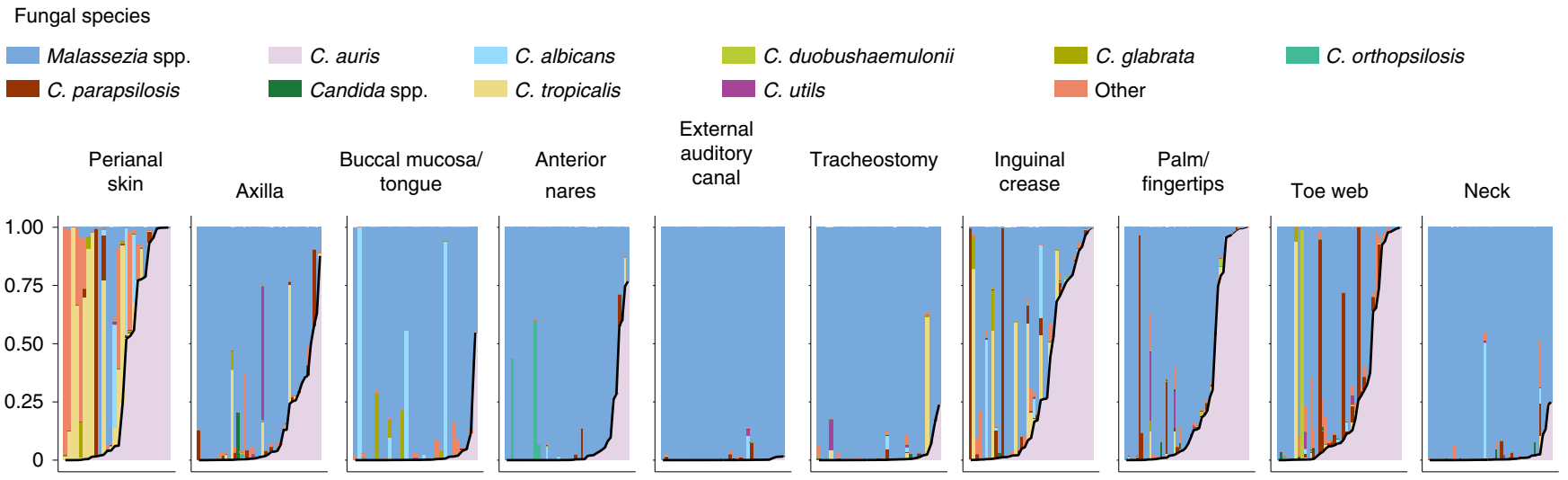

b

Bacterial phylum

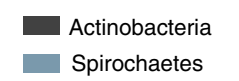

Bacteroidetes
Synergistetes

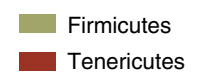

Fusobacteria

Verrucomicrobia
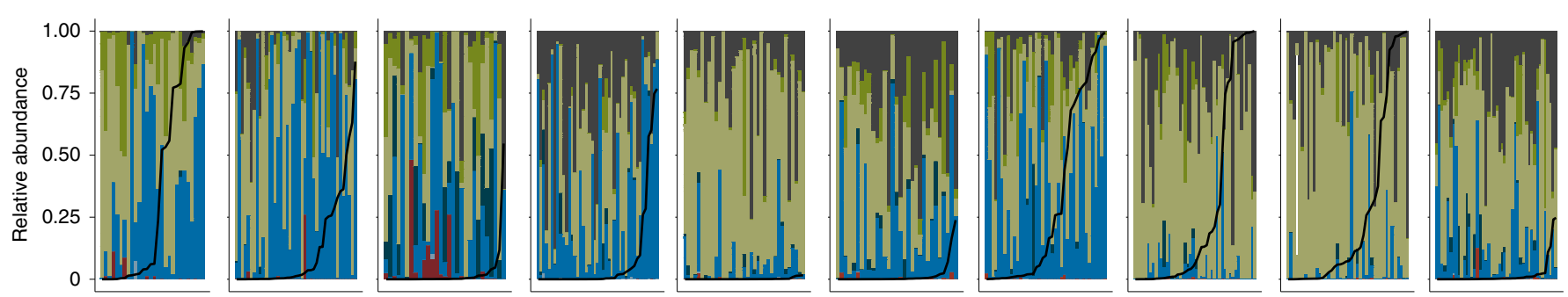

c

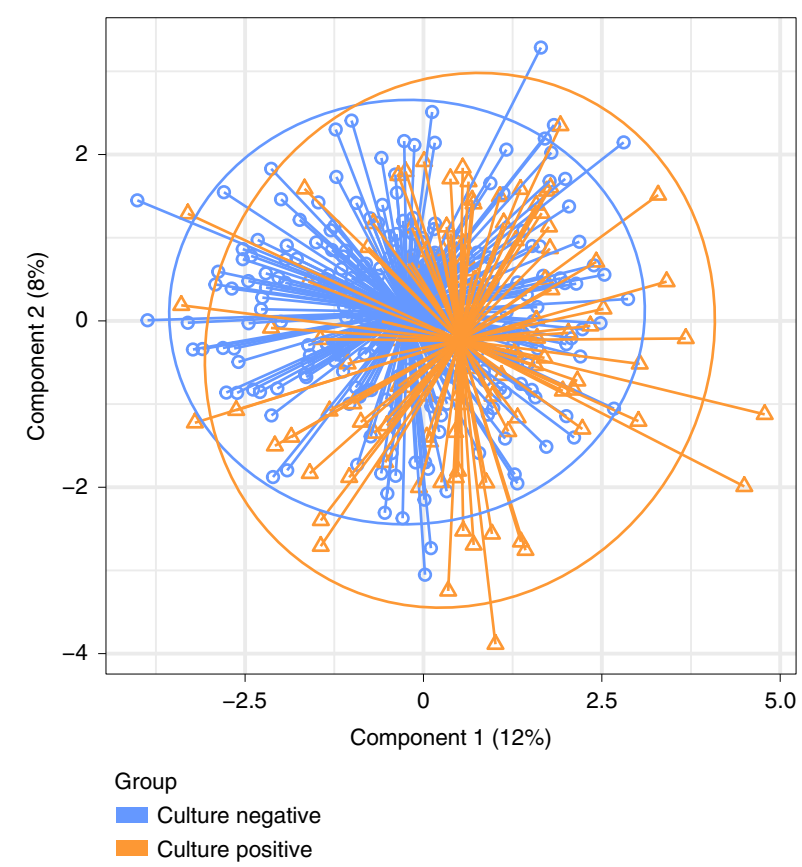

d

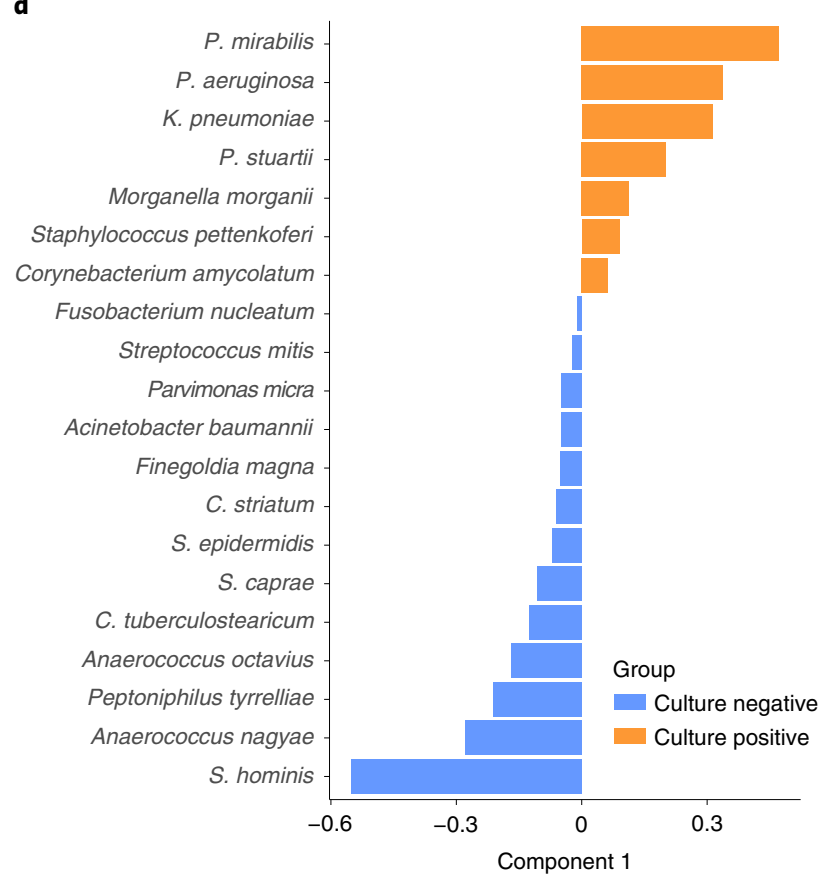

Fig. 3 | Underlying skin microbiome (fungal and bacterial communities) integrated with C. auris-colonization status. Panels represent the body-site-specific relative abundance of fungal (a) or bacterial (b) organisms. Bars represent the 51 participants, ordered by $C$. auris abundance. The inner black curve represents the relative abundance of $\boldsymbol{C}$. auris for each sample for each participant. $\mathbf{a}$, Relative abundance of fungal species at each body site surveyed for each participant. Colors correspond to fungal genera or individual Candida spp. Genera included in the 'other' category include Saccharomyces, Trichosporon, Trichophyton and Aspergillus. b, Relative abundance of bacteria, colored by phylum, reveals site-specific associations of $C$. auris with Proteobacteria. c,d, Colors correspond to sample or loadings associated with culture negativity or positivity. $\mathbf{c}$, Sample projection across first and second components of sPLS-DA. d, Variable loadings across the first sPLS-DA component. 


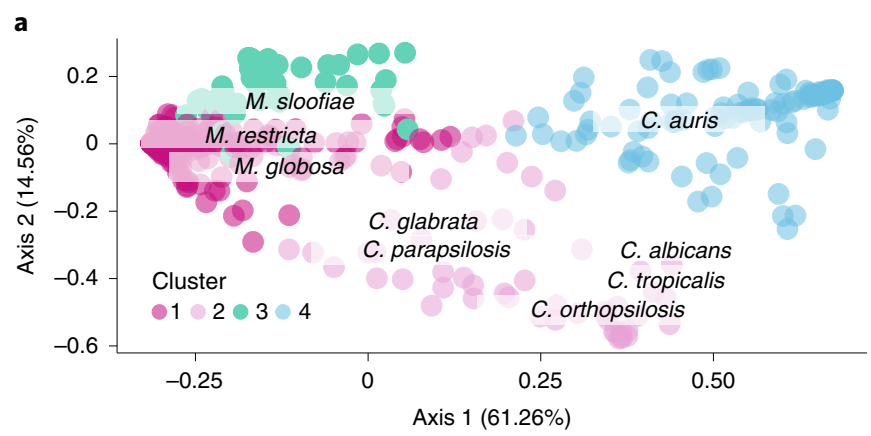

b

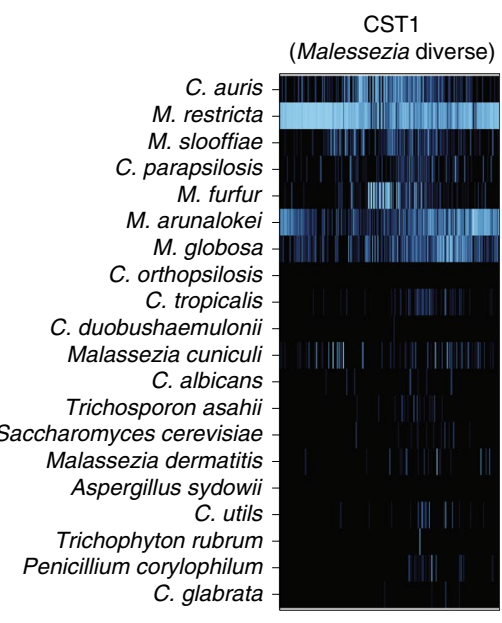

c

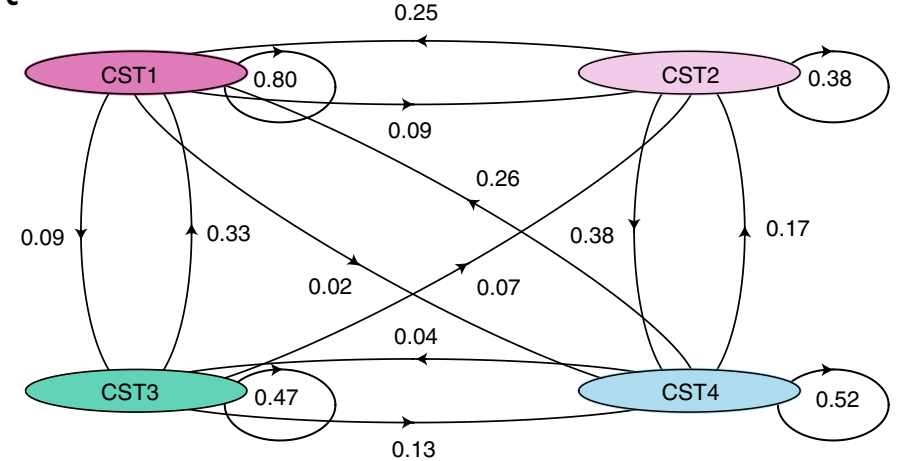

CST2

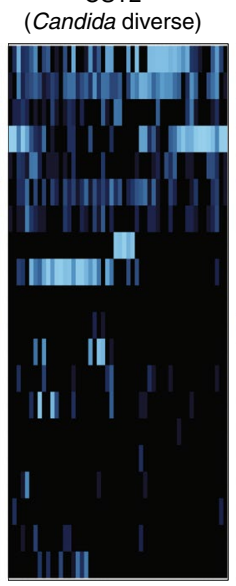

CST4

(C. auris)
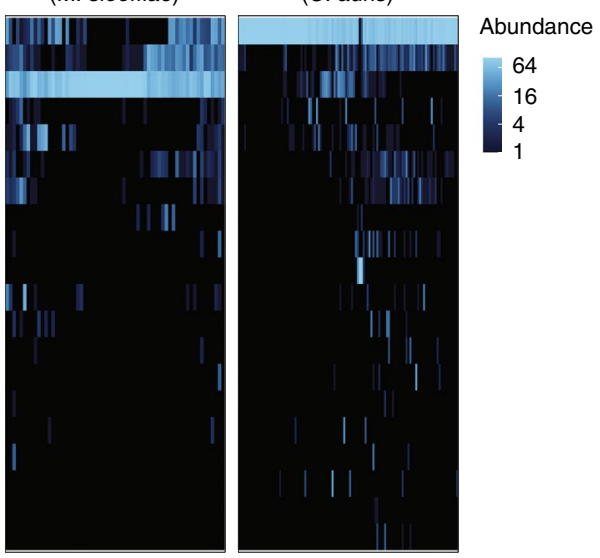

d

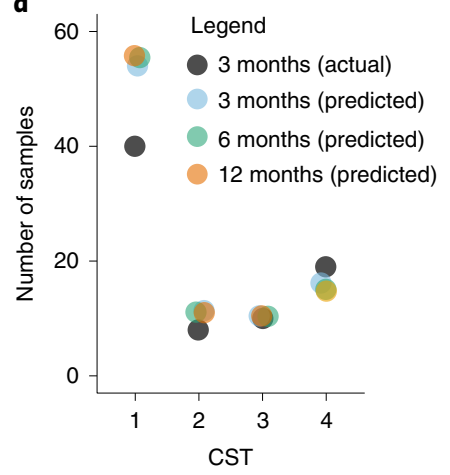

Fig. 4 | Skin fungal communities, dominated by Malassezia and Candida species, have differential stability, resilience and likelihood of invasion by C. auris. a, Principal-coordinate analysis of the weighted UniFrac metric of the fungal community at each body site (toe webs, fingertips and/or palm, inguinal crease, anterior nares). Samples are shaded according to CST identity, as revealed by 'partition around medoids' analysis. CST1 tends to be dominated by $M$. restricta $(N=256,53.0 \%)$, CST2 by a variety of Candida species $(N=52,10.7 \%)$, CST3 by M. slooffiae ( $N=62,12.8 \%)$ and CST4 by C. auris $(N=113,23.4 \%)$. Segregation of Malassezia and Candida species across the first axis explains $61 \%$ of the variance. Candida species segregate across the second major axis, which accounts for $~ 15 \%$ of the variance. $\mathbf{b}$, Relative abundance of the top 20 species in each sample, clustered by CST. Shading is based on the relative abundance of taxa within each sample. c, Self and interstate transition probabilities inferred for samples of the toe webs, palm and/or fingertips, inguinal crease and anterior nares. d, Scatterplot of the predicted numbers of samples in each CST at 3, 6 and 12 months after sample collection compared to the actual number of samples at 3 months. Predictions were generated using the Markov chain in c.

account both phylogeny and relative abundance of taxa. Cluster analysis revealed four clusters or fungal community state types (CSTs) in the data (Extended Data Fig. 6). Predominance of either Malassezia (CST1, CST3) or Candida species (CST2, CST4) drove segregation of samples along the first major axis, which explained $61.3 \%$ of the variation (Fig. 4a). While genus-level differences defined axis 1, species-level variation drove segregation of communities along axis 2 , which accounted for $14.6 \%$ of the variation.

CST1 best approximates a typical healthy skin commensal community ${ }^{25}$, composed predominantly of Malassezia restricta and including other commensal Malassezia species: Malassezia globosa, Malassezia furfur and Malassezia arunalokei (Fig. 4b). CST2 was diverse with inclusion of multiple Candida spp., including C. auris, C. albicans, C. tropicalis, C. parapsilosis, Candida orthopsilosis and C. metapsilosis. The remaining CSTs were less diverse. CST3 was predominated by Malassezia slooffiae, with frequent high-abundance co-colonization by either C. auris or M. restricta. Most samples in CST4 were mono-predominated by $C$. auris, although a few samples were co-predominated by M. restricta or C. parapsilosis. 
Next, we investigated mycobiome dynamics, using data from the first two surveys to model the probability that an individual body site would transition between CSTs (Fig. 4c). Malassezia-diverse CST1 was the most stable state with the highest self-transition ('remain the same') probability $(P=0.82)$. By contrast, CST2, with diverse Candida species, not typically abundant on healthy human skin, comprised the least stable community. CST2 samples were as likely to transition to CST4 (predominated by C. auris) as they were to remain as CST2 $(P=0.38)$. By contrast, Malassezia-diverse CST1 samples rarely transitioned toward $C$. auris-predominant CST4 $(P=0.02)$, with higher transition frequency toward the diverse Candida CST2 $(P=0.07)$ or $M$. slooffiae-dominated CST3 $(P=0.09)$. While $M$. slooffiae, $C$. tropicalis and C. parapsilosis ${ }^{25-27}$ rarely dominate the skin microbiome of healthy volunteers, they are often found at low abundance and low prevalence in skin communities. In sum, these data suggest potential value in monitoring or intervening at the intermediate state when the skin community migrates away from the diverse commensal Malassezia community.

To gain further insight into the stability of $C$. auris colonization, we used a Markov chain built from the first two point prevalence surveys to predict the number of samples in each CST at the third-point prevalence survey and to forecast patterns at time points beyond the study interval. This model predicts $C$. auris-colonization prevalence at the third-point prevalence survey (Fig. $4 \mathrm{~d}$ ) and forecasts persistent $C$. auris skin colonization across multiple sites for over a year. Persistent colonization is consistent with findings from our culture data, indicating that $C$. auris stably colonizes human skin, exhibiting particularly stable colonization at the nares (Extended Data Fig. 7).

Over the course of the study, nine residents who were initially colonized at one or more body sites cleared $C$. auris colonization across all body sites by the end of the study. For these nine residents, the proportion of samples dominated by C. auris (CST4) decreased from $16.0 \%$ to $0 \%$ (CST4) from the first to the third time point, while the proportion of sites dominated by Malassezia species (CST1) increased from $68.0 \%$ to $79.3 \%$, respectively (Extended Data Fig. 8). In individuals who were persistently colonized, by contrast, the proportion of samples dominated by $C$. auris remained roughly constant over time $(\sim 30 \%)$. Communities that shifted away from C. auris toward Malassezia predominance experienced an increase in mycobiome diversity (Extended Data Fig. 9) and a reduction in proteobacterial abundances among the bacterial community in concert with the loss of $C$. auris. Clinically, transiently colonized residents were less likely to require mechanical ventilation or gastrostomy tubes than residents who were persistently colonized $\left(\chi^{2}, P<0.05\right)$.

Collectively, these data suggest the resilience of the commensal skin communities to initial invasion. However, once $C$. auris establishes predominance in a community, it is capable of establishing persistent colonization of the skin.

\section{Discussion}

Here we integrate genomic, clinical and epidemiologic data, analyzing almost 4,000 samples from 57 residents over a 3-month period to investigate factors underlying persistent $C$. auris colonization of residents of the ventilator ward of a skilled nursing facility with endemic C. auris and routine CHG bathing. Our investigation provides insights that can be applied to the design of potentially more effective programs to control C. auris transmission and prevent infections in long-term care settings, which bear a disproportionate burden of C. auris disease.

Colonization of sites not screened routinely may facilitate healthcare-associated transmission ${ }^{17}$. Screening of residents at high risk of $C$. auris carriage is typically carried out by collecting a composite bilateral axillae and inguinal creases swab sample. In our study, unilateral screening of axilla and inguinal crease would have detected only 35 of 57 colonized residents; we found that screening of six body sites was required to identify all colonized residents. The nares and palms and/or fingertips were colonized more frequently and carried higher bioburdens than other sites. While the inguinal creases and axillae were identified by Public Health England to be the two most persistently positive $\operatorname{sites}^{18}$, a study conducted by the New York State Department of Public Health reported the sensitivity of anterior nares to be higher than that of composite axillae and inguinal creases ${ }^{28}$. Quantitative culture results of South Asian clade I from New York State and those of South American clade IV in our study are concordant, demonstrating higher bioburden in anterior nares than in axillae and/or inguinal creases for C. auris-colonized residents. Because sampling six body sites may not be practical for routine screening, targeting high-yield sites such as axillae and/or inguinal creases and anterior nares is still recommended. Modifying screening strategies to include additional body sites during intractable outbreaks may permit identification of reservoirs for intrafacility transmission. Investigating innovative specimen collection methods, such as using sponge swabs to sample larger areas of skin ${ }^{29}$, may also be of benefit. Frequent and dense colonization of anterior nares and palms and/or fingertips suggests that nasal decolonization and promotion of resident hand hygiene may be options to help reduce cross-transmission of $C$. auris in skilled nursing facilities. Finally, the imperfect sensitivity of most screening methods highlights the importance of universal, facility-wide infection-prevention measures, such as healthcare provider hand hygiene and environmental disinfection, in reducing $C$. auris transmission.

Despite the apparent susceptibility of $C$. auris isolates to $\mathrm{CHG}$ in vitro, the odds of recovering $C$. auris from skin sites by culture were reduced only at high measured concentrations of CHG. It is notable that the nares, a site not subject to CHG bathing, and the body sites with the lowest average concentration of CHG (palms and/or fingertips and toe webs) were frequently and densely colonized with C. auris. Heterogeneity in CHG concentration may have been due to differences in time since the last CHG bath, a variable not recorded by ventilator-capable skilled nursing facility personnel. Relatively high concentrations of $\mathrm{CHG}$ observed in inguinal samples may result from increased focal bathing as part of incontinence care. Nevertheless, our finding that only $7.3 \%$ of samples had concentrations of CHG sufficiently high to be associated with reduced odds of $C$. auris colonization suggests that a point of intervention may be ensuring that all skin sites maintain high residual concentrations of $\mathrm{CHG}^{10}$. We were encouraged to find no evidence of an association between $\mathrm{CHG}$ concentration and perturbation of the commensal skin microbiome.

Persistent $C$. auris colonization reported here is consistent with observations of several patient and resident populations in acute and long-term care settings, respectively ${ }^{3,18}$, including another facility with routine $\mathrm{CHG}$ bathing 9 . Our analysis of mycobiome dynamics suggests that commensal, Malassezia-predominated communities are resilient to invasion by $C$. auris. By contrast, we observed that C. auris predominance is relatively stable once it occurs, with a $30-50 \%$ chance that $C$. auris will persist at sites once it comes to dominate. The ascension of $C$. auris to dominance in the community appeared to occur through an intermediate state (CST2) in which diverse Candida spp. co-predominate skin sites with a $\sim 40 \%$ probability that sites would transition from the diverse Candida state toward C. auris predominance. This finding is consistent with prior work demonstrating that C. auris has a higher predilection for skin colonization than does C. albicans ${ }^{6,30}$, which in turn is a better skin colonizer than C. tropicalis or C. parapsilosis ${ }^{26}$.

Our observation that a community of diverse Candida species is an intermediate state between commensal Malassezia and C. auris predomination provides an additional possible point of intervention for infection prevention. While we did not examine the in vitro susceptibility of Malassezia spp. to CHG, microbiome sequencing results failed to provide evidence of a linear relationship 
between CHG concentration and the abundance of Malassezia spp. Selection of $C$. auris by CHG bathing is one possible explanation for the high transition rate to $C$. auris predominance. However, countering this argument is our observation that $C$. auris was more stable at the nares, a site not subjected to CHG bathing, as compared to sites undergoing bathing. Our analysis revealed a variety of Gram-negative bacterial species commonly found in nursing home settings, including $P$. aeruginosa, $P$. mirabilis, $K$. pneumoniae and P. stuartii, to be enriched in C. auris-positive samples. However, due to the widespread prevalence of $C$. auris in the facility at the outset of the study and complex medical histories of these residents, we could not determine whether the bloom of Gram-negative species preceded C. auris colonization or vice versa, nor could we determine the temporal relationship between antibiotic receipt and colonization with these Gram-negative bacteria or C. auris. Similarly, our study design precluded determination of whether associations of $C$. auris colonization with other clinical covariates, such as mechanical ventilation, reflect merely the generally poorer health of colonized residents or are causally related to colonization. Elucidating the effect of antibiotic exposure on skin microbial communities and $C$. auris colonization and dominance is of particular importance, as this risk factor may be modifiable. Exposure to a carbapenem, vancomycin or fluconazole was associated with increased odds of $C$. auris colonization in another recent study of long-term care residents ${ }^{31}$.

One limitation of this study is that the different clades of C. auris may exhibit different body-site tropisms; therefore, our results may not be generalizable to colonization with $C$. auris other than South American clade IV. The single-center setting of our study also may reduce its generalizability. Sample collection for this work began after prevalence at the facility reached saturation, making it difficult to understand patterns of diversity in the microbiome before the introduction of $C$. auris. To gain insight into how antibiotic-mediated reductions in colonization resistance might increase risk of colonization, future work should aim to disambiguate the onset of these effects in other healthcare settings. Finally, our experimental design enabled quantification of $\mathrm{CHG}$ concentrations across body sites but lacked a comparator group against which we could assess the impact of CHG bathing as a controlled intervention.

Our data suggest that residents of ventilated skilled nursing facilities where C. auris is endemic and CHG bathing is routine have highly personalized patterns of $C$. auris skin colonization. This site-to-site variability in colonization limits infection-control strategies predicated on targeting only known C. auris-colonized individuals; facility or unit-wide infection-control approaches may be more effective. We found that perturbation of the skin microbiome is a potential risk factor for $C$. auris colonization and persistence. These data have implications not only for facilities where C. auris is endemic but also for other facilities embedded within the same regional healthcare networks ${ }^{32}$. While C. auris outbreaks in the United States have been reported primarily in long-term care settings, a recent report of a $C$. auris outbreak in a coronavirus disease 2019 (COVID-19) specialty care unit highlights the threat that C. auris poses to acute care hospitals ${ }^{33}$. Our findings have identified candidate targets for future interventions related to skin microbiome dynamics, including the potential for preserving or restoring commensal skin microbiota to augment existing approaches for C. auris control.

\section{Online content}

Any methods, additional references, Nature Research reporting summaries, source data, extended data, supplementary information, acknowledgements, peer review information; details of author contributions and competing interests; and statements of data and code availability are available at https://doi.org/10.1038/ s41591-021-01383-w.
Received: 29 October 2020; Accepted: 5 May 2021;

Published online: 21 June 2021

\section{References}

1. Jeffery-Smith, A. et al. Candida auris: a review of the literature. Clin. Microbiol. Rev. 31, e00029-17 (2018).

2. Tsay, S. et al. Notes from the field: ongoing transmission of Candida auris in health care facilities-United States, June 2016-May 2017. MMWR Morb. Mortal. Wkly Rep. 66, 514-515 (2017).

3. Vallabhaneni, S. et al. Investigation of the first seven reported cases of Candida auris, a globally emerging invasive, multidrug-resistant fungusUnited States, May 2013-August 2016. Am. J. Transplant. 17, 296-299 (2017)

4. Chen, J. et al. Is the superbug fungus really so scary? A systematic review and meta-analysis of global epidemiology and mortality of Candida auris. BMC Infect. Dis. 20, 827 (2020).

5. Centers for Disease Control and Prevention. Antibiotic Resistance Threats in the United States, 2019 (US Department of Health and Human Services, CDC, 2019).

6. Horton, M. V. et al. Candida auris forms high-burden biofilms in skin niche conditions and on porcine skin. mSphere 5, e00972-19 (2020).

7. Weschler, C. J. et al. Squalene and cholesterol in dust from Danish homes and daycare centers. Environ. Sci. Technol. 45, 3872-3879 (2011).

8. Biswal, M. et al. Controlling a possible outbreak of Candida auris infection: lessons learnt from multiple interventions. J. Hosp. Infect. 97, 363-370 (2017).

9. Schelenz, S. et al. First hospital outbreak of the globally emerging Candida auris in a European hospital. Antimicrob. Resist. Infect. Control 5, 35 (2016).

10. Supple, L. et al. Chlorhexidine only works if applied correctly: use of a simple colorimetric assay to provide monitoring and feedback on effectiveness of chlorhexidine application. Infect. Control Hosp. Epidemiol. 36, 1095-1097 (2015).

11. Weiner, L. M., Webb, A. K., Walters, M. S., Dudeck, M. A. \& Kallen, A. J. Policies for controlling multidrug-resistant organisms in US healthcare facilities reporting to the National Healthcare Safety Network, 2014. Infect. Control Hosp. Epidemiol. 37, 1105-1108 (2016).

12. Pacilli, M. et al. Regional emergence of Candida auris in Chicago and lessons learned from intensive follow-up at one ventilator-capable skilled nursing facility. Clin. Infect. Dis. 71, e718-e725 (2020).

13. Adams, E. et al. Candida auris in healthcare facilities, New York, USA, 2013-2017. Emerg. Infect. Dis. 24, 1816-1824 (2018).

14. Lockhart, S. R. et al. Simultaneous emergence of multidrug-resistant Candida auris on 3 continents confirmed by whole-genome sequencing and epidemiological analyses. Clin. Infect. Dis. 64, 134-140 (2017).

15. Larkin, E. et al. The emerging pathogen Candida auris: growth phenotype, virulence factors, activity of antifungals, and effect of SCY-078, a novel glucan synthesis inhibitor, on growth morphology and biofilm formation. Antimicrob. Agents Chemother. 61, e02396-16 (2017).

16. Ostrowsky, B. et al. Candida auris isolates resistant to three classes of antifungal medications-New York, 2019. MMWR Morb. Mortal. Wkly Rep. 69, 6-9 (2020).

17. Snitkin, E. S. et al. Tracking a hospital outbreak of carbapenem-resistant Klebsiella pneumoniae with whole-genome sequencing. Sci. Transl. Med. 4, 148ra116 (2012).

18. Bishop, L. et al. Public Health England: Guidance for the Laboratory Investigation, Management and Infection Prevention and Control for Cases of Candida auris v2.0 https://www.gov.uk/government/publications/ candida-auris-laboratory-investigation-management-and-infectionprevention-and-control (2017)

19. Chow, N. A. et al. Tracing the evolutionary history and global expansion of Candida auris using population genomic analyses. mBio 11, e03364-19 (2020)

20. Centers for Disease Control and Prevention, National Center for Emerging and Zoonotic Infectious Diseases. Screening for Candida auris Colonization https://www.cdc.gov/fungal/candida-auris/c-auris-screening.html (accessed 29 May 2020).

21. Tsay, S., Kallen, A., Jackson, B. R., Chiller, T. M. \& Vallabhaneni, S. Approach to the investigation and management of patients with Candida auris, an emerging multidrug-resistant yeast. Clin. Infect. Dis. 66, 306-311 (2018).

22. Sherry, L. et al. Biofilm-forming capability of highly virulent, multidrug-resistant Candida auris. Emerg. Infect. Dis. 23, 328-331 (2017).

23. Moore, G., Schelenz, S., Borman, A. M., Johnson, E. M. \& Brown, C. S. Yeasticidal activity of chemical disinfectants and antiseptics against Candida auris. J. Hosp. Infect. 97, 371-375 (2017).

24. Popovich, K. J. et al. Relationship between chlorhexidine gluconate skin concentration and microbial density on the skin of critically ill patients bathed daily with chlorhexidine gluconate. Infect. Control Hosp. Epidemiol. 33, 889-896 (2012)

25. Findley, K. et al. Topographic diversity of fungal and bacterial communities in human skin. Nature 498, 367-370 (2013). 
26. Raz-Pasteur, A., Ullmann, Y. \& Berdicevsky, I. The pathogenesis of Candida infections in a human skin model: scanning electron microscope observations. ISRN Dermatol. 2011, 150642 (2011).

27. Prohic, A., Jovovic Sadikovic, T., Krupalija-Fazlic, M. \& Kuskunovic-Vlahovljak, S. Malassezia species in healthy skin and in dermatological conditions. Int. J. Dermatol. 55, 494-504 (2016).

28. Zhu, Y. et al. Laboratory analysis of an outbreak of Candida auris in New York from 2016 to 2018: impact and lessons learned. J. Clin. Microbiol. 58, e01503-1 (2020).

29. Nutman, A. et al. Detecting carbapenem-resistant Acinetobacter baumannii (CRAB) carriage: which body site should be cultured? Infect. Control Hosp. Epidemiol. 41, 965-967 (2020).

30. Huang, X. et al. Murine model of colonization with fungal pathogen Candida auris to explore skin tropism, host risk factors and therapeutic strategies. Cell Host Microbe 29, 210-221 (2020).
31. Rossow, J. et al. Factors associated with Candida auris colonization and transmission in skilled nursing facilities with ventilator units, New York, 2016-2018. Clin. Infect. Dis. https://doi.org/10.1093/cid/ciaal462 (2020).

32. Snitkin, E. S. et al. Integrated genomic and interfacility patient-transfer data reveal the transmission pathways of multidrug-resistant Klebsiella pneumoniae in a regional outbreak. Sci. Transl. Med. 9, eaan0093 (2017).

33. Prestel, C. et al. Candida auris outbreak in a COVID-19 specialty care unit-Florida, July-August 2020. MMWR Morb. Mortal. Wkly Rep. 70 , 56-57 (2021).

Publisher's note Springer Nature remains neutral with regard to jurisdictional claims in published maps and institutional affiliations.

This is a U.S. government work and not under copyright protection in the U.S.; foreign copyright protection may apply 2021

\section{NISC Comparative Sequencing Program}

Jim Mullikin ${ }^{4}$, Jim Thomas ${ }^{4}$, Alice Young ${ }^{4}$, Gerry Bouffard ${ }^{4}$, Betty Barnabas ${ }^{4}$, Shelise Brooks ${ }^{4}$, Joel Han ${ }^{4}$, Shi-ling $\mathrm{Ho}^{4}$, Juyun Kim${ }^{4}$, Richelle Legaspi ${ }^{4}$, Quino Maduro ${ }^{4}$, Holly Marfani ${ }^{4}$, Casandra Montemayor ${ }^{4}$, Nancy Riebow ${ }^{4}$, Karen Schandler ${ }^{4}$, Brian Schmidt ${ }^{4}$, Christina Sison ${ }^{4}$, Mal Stantripop ${ }^{4}$, Sean Black ${ }^{4}$, Mila Dekhtyar ${ }^{4}$, Cathy Masiello ${ }^{4}$, Jenny McDowell ${ }^{4}$, Morgan Park ${ }^{4}$, Pam Thomas ${ }^{4}$ and Meg Vemulapalli ${ }^{4}$ 


\section{Methods}

Participant recruitment. This research complies with all relevant ethical human research regulations. The study was reviewed and approved by the Rush University Institutional Review Board, which granted expedited review and waiver of informed consent. Study participants did not receive monetary compensation for their participation. Demographics of the participant population are reported in Table 1 .

Statistics and reproducibility. We conducted an observational, serial cross-sectional study of residents of a ventilator-capable skilled nursing facility (vSNF) in Chicago, IL, USA. All residents on the 70-bed ventilator ward of the 300-bed vSNF at the time of the first survey were eligible for study participation. Although this work was exploratory, we conducted an a priori power analysis to ensure that we would have sufficient power to detect notable differences in the prevalence of $C$. auris colonization at different body sites, that is, to test the null hypothesis of uniform distribution of $C$. auris across body sites. When we were planning the study, we anticipated participation of 35 residents with $C$. auris colonization and 25 residents without $C$. auris colonization during the three serial point prevalence surveys. We based these estimates on documented incidence, prevalence and turnover rates at that time and an assumption of $80 \%$ resident participation. Assuming participation of 35 C. auris-colonized residents, true positive rates at the reference site ranging from $70 \%$ to $90 \%$ and $\alpha=0.05$, we calculated that we would have $80 \%$ power to detect relative risk in any comparison site ranging from 0.62 to 0.75 , that is, $44-68 \%$. In fact, we identified 45 C. aurispositive residents and $12 \mathrm{C}$. auris-negative residents during the first survey. Therefore, rather than seeking new residents to test during each of the subsequent two surveys, we continued to follow the original 57-resident cohort, eventually identifying a total of 49 residents who were ever positive for C. auris and eight residents who were never positive for $C$. auris.

Statistics on clinical variables describing the study cohort. Odds ratios and two-tailed $P$ values for continuous variables (that is, length of stay before the first swab, age, Charlson comorbidity index and Braden score) were determined using binary logistic regression analyses with $P$ values determined by Wald $\chi^{2}$ statistics. For dichotomous variables, two-tailed $P$ values were determined from Fisher's exact tests. To calculate an odds ratio for mechanical ventilation and antifungal receipt in the prior $90 \mathrm{~d}$, where the values in the 'not colonized' group are 0 , logit estimates were used, in which a correction of 0.5 was added to each cell in the table. None of the $P$ values for clinical analyses were adjusted for multiple comparisons.

Estimating the proportion of colonized residents by body sites. The proportion of colonized residents was calculated as previously described ${ }^{34}$. Exact binomial $95 \%$ confidence intervals were calculated using the 'epi.tests' function in the 'epiR' package.

Estimating sensitivity of sites for identifying colonized residents. We used a custom function leveraging the 'combn' function in base $\mathrm{R}$ and the 'epiR' package to generate the 'proportion of colonized individuals' for all possible two-way $(n=45)$, three-way $(n=120)$, four-way $(n=210)$, five-way $(n=252)$, six-way $(n=210)$, seven-way $(n=120)$, eight-way $(n=45)$ and nine-way $(n=10)$ groupings of the ten body sites. Sensitivity was computed by dividing the 'proportion of colonized individuals' by the proportion of residents positive at any body site.

Estimating bioburden of $\boldsymbol{C}$. auris across body sites. Flocked swabs (FLOQSwabs, Copan) were used to sample a $5 \times 5 \mathrm{~cm}^{2}$ area from each body site and were placed immediately in Amies medium with neutralizer without ether sulfate ${ }^{35,36}$. Aliquots $(100 \mu \mathrm{l})$ were inoculated directly onto CHROMagar Candida plates (Becton Dickinson) and incubated at $37^{\circ} \mathrm{C}$ for $7 \mathrm{~d}$. A second $100-\mu$ l aliquot was inoculated into Salt Sabouraud Dulcitol Broth and incubated at $40^{\circ} \mathrm{C}$ for $7 \mathrm{~d}$; cloudy broth cultures were subsequently inoculated onto CHROMagar Candida plates ${ }^{37}$ and incubated for $7 \mathrm{~d}$. Each unique colony morphology underwent identification to the species level by MALDI-TOF (VITEK MS Plus (bioMérieux). Resulting CFU from directly inoculated plates for all colonized sites (excluding uncolonized sites) at the first time point were plotted with the 'geom_density_ridges' function in the package 'ggridges'.

Axilla, inguinal crease and anterior nare samples were also processed with an MPN method that tested eight serial dilutions of the original sample with the enrichment broth method. Three replicates were performed for each dilution, enabling calculation of an MPN as described at https://www. fda.gov/food/laboratory-methods-food/bam-appendix-2-most-probabl e-number-serial-dilutions. High-throughput-format MPN analysis was performed using 96-well 2-ml deep-well blocks in which $500-\mu$ l volumes of Sabouraud broth with dulcitol, chloramphenicol and gentamicin were inoculated with $50-\mu \mathrm{l}$ sample aliquots from each replicate for each dilution. Culture blocks were incubated at $40^{\circ} \mathrm{C}$ for $7 \mathrm{~d}$ before plating onto CHROMagar Candida plates in $10-\mu \mathrm{l}$ volumes. Plates were checked for growth after $7 \mathrm{~d}$. Growth with morphology characteristic of $C$. auris was confirmed for both culture methods to the species level using MALDI-TOF.
CHG and antifungal susceptibility testing and determination of the CHG concentration on skin. To determine the CHG concentration on skin, swabs (Bio-Swab, Arrowhead Forensics) moistened with sterile water were used to sample areas of skin adjacent to those areas that were sampled for culture and microbiome analysis, only at sites bathed routinely with CHG. CHG concentrations were measured using a quantitative colorimetric method that was read by visual inspection as described previously ${ }^{38}$.

CHG susceptibility was determined using a yeast broth microdilution method in accordance with CLSI guidelines ${ }^{39,40}$, starting with a $20 \%$ solution of chlorhexidine digluconate (Sigma). Susceptibility to selected antifungal agents was determined using a commercial microdilution method (Sensititre YeastOne YO9 AST Plate, Thermo Scientific) and applying tentative breakpoints published by the $\mathrm{CDC}^{41}$.

\section{Association of CHG concentration with C. auris colonization. Mixed-effects} regression models were used to control for clustering of body sites within residents over time. For CHG concentration by body site, a mixed-effects ordinal logistic regression model was used. For testing the relation between CHG concentration $\left(\log _{2}\right.$ transformed) and C. auris, a series of mixed-effects binary logistic regression models was used. The first model tested the linear relation between CHG concentration and C. auris colonization ( $\log _{2}$ transformed), controlling for body site. This was followed by a series of ten models testing cutoff points for $\mathrm{CHG}$ concentration at each successive increment in the semiquantitative test for $\mathrm{CHG}$ concentration.

\section{Microbiome sample collection, DNA extraction, PCR amplification and} sequencing. Swab samples from each anatomic site were collected (Extended Data Fig. 1) using our previously published protocols; negative controls were also collected and subsequently processed in parallel with true samples as described previously $^{42}$. Briefly, foam swabs (Puritan, 2515061PF) were premoistened with Yeast Cell Lysis solution (Lucigen) and used to collect swab samples from the skin across individual sites that were adjacent to sites sampled for culture and CHG-concentration measurement. Swabs were stored in lysis solution at $-80^{\circ} \mathrm{C}$ following collection. For extraction, skin swabs were incubated in Yeast Cell Lysis buffer and Ready-Lyse Lysozyme solution (Lucigen) for $1 \mathrm{~h}$ with shaking at $37^{\circ} \mathrm{C}$. Steel beads $(5 \mathrm{~mm})$ were added to mechanically disrupt fungal cell walls using a TissueLyser (Qiagen) for $2 \mathrm{~min}$ at $30 \mathrm{~Hz}$, followed by 30 -min incubation at $65^{\circ} \mathrm{C}$ for complete lysis. MPC reagent was added to samples, and resulting supernatants were processed using the PureLink Genomic DNA kit (Invitrogen). DNA was eluted in DNA-Free PCR Water (Qiagen).

The ITS1 region was amplified using primers modified with Illumina adaptors 18S-F (5'-GTAAAAGTCGTAACAAGGTTTC) and 5.8S-1R (5'-GTTCAAAGAYTCGATGATTCAC) as previously described ${ }^{43,44}$. The following PCR conditions were used: $2.5 \mu \mathrm{l} 10 \times$ PCR buffer, $4 \mu \mathrm{l}$ dNTP mix, $0.25 \mu \mathrm{l}$ Takara LA Taq Polymerase (Clontech), $1 \mu \mathrm{l} 18 \mathrm{~S}-\mathrm{F}(10 \mu \mathrm{M}), 1 \mu \mathrm{l} 5.8 \mathrm{~S}-1 \mathrm{R}(10 \mu \mathrm{M})$, 13.75 $\mu$ l PCR Water (Qiagen) and 2.5 $\mu$ l DNA. Reactions were performed in duplicate for 30 cycles, combined, purified using the Agencourt AMPure XP system (Beckman Coulter) and quantified using the Quant-iT dsDNA kit (Invitrogen). Equivalent amounts of amplicons were pooled together, purified with the MinElute PCR purification kit (Qiagen) and sequenced on an Illumina MiSeq. The V1-V3 region of the bacterial $16 \mathrm{~S}$ gene was amplified under similar conditions using primers 27F (5'-AGAGTTTGATCCTGGCTCAG) and 534R (5'-ATTACCGCGGCTGCTGG).

Demultiplex and quality filtering of amplicon tables. A total of $5.3 \times 10^{7} \mathrm{raw}$ reads were analyzed for $16 \mathrm{~S}$ amplicons, while a total of $1.47 \times 10^{7}$ raw reads were analyzed for the ITS1 table. Forward and reverse reads were independently demultiplexed, and sequences were parsed into sample-specific files with Illumina's 'bcl2fastq' version 2.20 software; primers were trimmed from sequences using cutadapt $2.10^{*}$ before import into R 3.6.1 for quality filtering with the R package 'dada2'; the 'filterAndTrim' function was used to truncate reads at the first instance of a quality score of 2 before elimination of reads with more than 0 ambiguous characters, a maximum expected error exceeding 2 or a length less than 50 nucleotides. Error rates were learned independently on filtered forward and reverse reads using default parameters before dereplication, inference of sequence-specific errors (with self-consistency set to true and pooling set to true) and elimination of problematic reads. Dereplicated and filtered forward sequences were subsequently merged with their paired-end reads before construction of an amplicon sequence variant (ASV) table. The 'removeBimeraDenovo' function of dada2 set to the 'consensus' method was used to filter chimeras from each ASV table. Taxonomic assignment was then performed down to the species level when possible using the dada2 implementation of the RDP Naive Bayesian Classifier 3 trained on the RefSeq database version 2.0 downloaded in May 2018 for bacterial reads and the UNITE 'sh_general_release_dynamic' database downloaded on 2 February 2019. Datasets generated as direct output from dada2 are referred to as 'merged_16s' and 'merged_ITS', and sequencing depths associated with these tables were 12,466,948 (16S) and 39,481,121 (ITS).

Quality control of the sequence tables. To deplete taxa enriched in extraction controls and therefore likely associated with contamination, a data subset was 
generated, consisting only of the technical controls (16S, $n=150$; ITS, $n=90$ ), which were sequenced in parallel with true samples (16S, $n=1,258$; ITS, $n=1,318$ ). An enrichment score was calculated for enrichment of taxa in controls compared to samples and vice versa; taxa enriched in controls relative to samples were deleted from the dataset as previously described ${ }^{45}$. Filtering using this method reduced the $16 \mathrm{~S}$ table to 854 taxa across 1,243 samples and the fungal table to 136 taxa across 1,226 samples. Reads were agglomerated to the taxonomic rank of species using the 'tax_glom' function of 'phyloseq'. Subsequent to taxonomic agglomeration, samples with fewer than 500 reads per sample were dropped. Finally, samples were dropped from the $16 \mathrm{~S}$ table if the ITS1 sample failed quality control, generating sample sets of equal size $(n=1,152)$ across 'bac_match' and 'its_match' datasets.

Identifying bacteria associated with colonization. To reduce the influence of highly variant taxa on findings, species present in fewer than $20 \%$ of 16 S samples were dropped. Reads were subsequently transformed using centered-log-ratio transformation as implemented in the 'compositions' package. The 'splsda' function of the 'mixomics' package was used to identify bacterial species positively or negatively associated with $C$. auris culture.

Community state type analysis. The weighted UniFrac statistic was computed on Malassezia and Candida species with the distance function of 'phyloseq' before performing independent rounds of cluster analysis from one to 30 clusters using the 'partition around medoids' function of the 'cluster' package. The gap statistic was computed for each cluster analysis from one to 30 using the 'clusGap' function and 1,000 Monte Carlo bootstrap samples. To evaluate the robustness of the identification of four clusters in the data, this process was repeated using the BrayCurtis dissimilarity metric. The four clusters for the weighted UniFrac analysis were subsequently labeled as CSTs.

Markov chains. A data subset was generated in which only samples from 23 residents who had a complete set of survey 1 and survey 2 samples at the fingertips, toe webs, nares and inguinal crease were retained. Based on prior work ${ }^{46}$, a custom function was written to generate the initial state matrices from the phyloseq S4 object 'its_match'. Transition matrices were obtained with a custom function that standardized each initial state matrix to four rows and four columns before normalization to row sums. Missing values, corresponding to states that were not observed at a given site, were replaced with 0 . Each transition matrix was subsequently used as input for the new 'markovchain' function of the 'markovchain' package. Predictions for $N$-month time points were obtained by multiplying the initial state matrix by the transition matrix raised to the $N$ th power. Each transition matrix was visualized using a custom function leveraging the 'plotmat' function in the 'diagram' package.

Illumina whole-genome sequencing. Isolates of C. auris (various clades) were streaked onto CHROMagar Candida plates (BD, 254093) and incubated at $37^{\circ} \mathrm{C}$ for $24-48 \mathrm{~h}$. Colonies were transferred to $600 \mu \mathrm{l}$ Yeast Cell Lysis buffer (Lucigen, MPY80200) with $3 \mu \mathrm{l}$ RNase (Qiagen, 19101) and 1-mm glass beads (MP Biomedicals, 116912050-CF). Cells were subjected to bead beating in a FastPrep 5G (MP Biomedicals) at speed 6.5 for $15 \mathrm{~s}$ and placed on ice for $1 \mathrm{~min}$, and the procedure was repeated. The cell lysate was incubated at $65^{\circ} \mathrm{C}$, and the protocol continued as directed (Lucigen, MPY80200). The genomic DNA pellet was resuspended in $0.1 \times \mathrm{TE}, \mathrm{pH}$ 8.0. Libraries were generated from genomic DNA with the Nextera XT Library Preparation kit (Illumina) as per the manufacturer's recommendations. Libraries were pooled at an equimolar ratio for sequencing on the Illumina NovaSeq 6000 to obtain a minimum of 2.6 million 151-bp reads per individual library. Illumina sequence data were processed using RTA version 3.4.4.

Nanopore sequencing and assembly. A fraction of genomic DNA prepared for lllumina whole-genome sequencing was reserved for nanopore sequencing and assembly. Genomic DNA was assessed by Femto Pulse (Agilent) analysis and determined to be approximately $15 \mathrm{~kb}$; therefore, shearing was not performed. A library was prepared for long-read Oxford Nanopore Technologies (ONT) sequencing from $1 \mu \mathrm{g}$ DNA using the Ligation Sequencing kit (ONT, SQK-LSK109). Twenty fmol of the library was sequenced on an R9 flow cell using the ONT GridION platform running MinKNOW 3.6.5 and run for $72 \mathrm{~h}$ with refueling at $48 \mathrm{~h}$. Guppy 3.6.0 was used to convert raw sequence data files to basecall fastq files. A total of $13.0 \mathrm{~Gb}$ of data was collected with an N50 length of $14.3 \mathrm{~kb}$ and a mean read length of $11.4 \mathrm{~kb}$. ONT sequence reads were randomly downsampled to an estimated $200 \times$ coverage using 'seqtk' version 1.2 (https:// github.com/lh3/seqtk) and assembled using Canu 2.0 (ref. ${ }^{47}$ ). Assembled contigs were polished with the ONT sequence reads for one round using Racon version 1.3.1 (ref. ${ }^{48}$ ), followed by two rounds with medaka version 1.0.3 (https://github. $\mathrm{com} /$ nanoporetech/medaka). Contigs were polished further by aligning Illumina short reads using Bowtie 2 version 2-2.4.2 (ref. ${ }^{49}$ ) and running Pilon version 1.23 (ref. ${ }^{50}$ ) on the alignment file.

Variant calling. Illumina NovaSeq reads for each of four outbreak isolate genomes were aligned to the nanopore reference genome, and single-nucleotide variants were identified with snippy version 4.4.1 (https:/github.com/tseemann/snippy) with default options deploying BWA version 0.7.17 and FreeBayes version 1.3.5 algorithms, requiring a read depth of at least $10 \times$ and the additional flag '-minfrac 0.9 ', requiring at least $90 \%$ of reads in support of the variant nucleotide call. SAMtools (version 1.11) coverage was used to estimate coverage and mapping quality across the genomes of isolates (mean coverage, 99.99; mean MAPQ score, 59.6).

Reporting Summary. Further information on research design is available in the Nature Research Reporting Summary linked to this article.

\section{Data availability}

Sequencing data are publicly available in the NIH Short Read Archive under BioProject accession number PRJNA672955. The RefSeq database version 2.0 downloaded on May 2018 and the UNITE 'sh_general_release_dynamic' database downloaded on 2 February 2019 were used to assign bacterial and fungal taxonomy, respectively, and can be found at https://github.com/skinmicrobiome/ cauris_colonization. Intermediate data files including the OTU table, the taxonomy table and the sample data mapping file are included in Supplementary Data 1.

\section{Code availability}

The $\mathrm{R}$ code used to generate figures is provided in Supplementary Data 2-5 and can be found at https://github.com/skinmicrobiome/cauris_colonization.

\section{References}

34. Thurlow, C. J. et al. Anatomic sites of patient colonization and environmental contamination with Klebsiella pneumoniae carbapenemase-producing Enterobacteriaceae at long-term acute care hospitals. Infect. Control Hosp. Epidemiol. 34, 56-61 (2013).

35. Reichel, M., Heisig, P. \& Kampf, G. Pitfalls in efficacy testing-how important is the validation of neutralization of chlorhexidine digluconate? Ann. Clin. Microbiol. Antimicrob. 7, 20 (2008).

36. Kampf, G. What is left to justify thÿe use of chlorhexidine in hand hygiene? J. Hosp. Infect. 70, 27-34 (2008).

37. Welsh, R. M. et al. Survival, persistence, and isolation of the emerging multidrug-resistant pathogenic yeast Candida auris on a plastic health care surface. J. Clin. Microbiol. 55, 2996-3005 (2017).

38. Edmiston, C. E. Jr. et al. Preoperative shower revisited: can high topical antiseptic levels be achieved on the skin surface before surgical admission? J. Am. Coll. Surg. 207, 233-239 (2008).

39. CLSI. Reference Method for Broth Dilution Antifungal Susceptibility Testing of Yeasts; Approved Standard-Third Edition (Clinical and Laboratory Standards Institute, 2008).

40. CLSI. CLSI document M27-A3 (Clinical and Laboratory Standards Institute, 2008).

41. Centers for Disease Control and Prevention, National Center for Emerging and Zoonotic Infectious Diseases (NCEZID), Division of Foodborne, Waterborne, and Environmental Diseases (DFWED). Antifungal Susceptibility Testing and Interpretation https://www.cdc.gov/fungal/ candida-auris/c-auris-antifungal.html (2020).

42. Oh, J. et al. Biogeography and individuality shape function in the human skin metagenome. Nature 514, 59-64 (2014).

43. Khot, P. D., Ko, D. L. \& Fredricks, D. N. Sequencing and analysis of fungal rRNA operons for development of broad-range fungal PCR assays. Appl. Environ. Microbiol. 75, 1559-1565 (2009).

44. Fadrosh, D. W. et al. An improved dual-indexing approach for multiplexed $16 \mathrm{~S}$ rRNA gene sequencing on the Illumina MiSeq platform. Microbiome 2, 6 (2014).

45. Proctor, D. M. et al. A spatial gradient of bacterial diversity in the human oral cavity shaped by salivary flow. Nat. Commun. 9, 681 (2018).

46. DiGiulio, D. B. et al. Temporal and spatial variation of the human microbiota during pregnancy. Proc. Natl Acad. Sci. USA 112, 11060-11065 (2015).

47. Koren, S. et al. Canu: scalable and accurate long-read assembly via adaptive $k$-mer weighting and repeat separation. Genome Res. 27, 722-736 (2017).

48. Vaser, R., Sovic, I., Nagarajan, N. \& Sikic, M. Fast and accurate de novo genome assembly from long uncorrected reads. Genome Res. 27, 737-746 (2017).

49. Langmead, B. \& Salzberg, S. L. Fast gapped-read alignment with Bowtie 2. Nat. Methods 9, 357-359 (2012).

50. Walker, B. J. et al. Pilon: an integrated tool for comprehensive microbial variant detection and genome assembly improvement. PLOS ONE 9, e112963 (2014).

\section{Acknowledgements}

The findings and conclusions in this report are those of the authors and do not necessarily represent the official position of the CDC. This work was supported in part by CDC contract number 75D30118C02900. This study used the computational resources of the National Institutes of Health (NIH) HPC Biowulf Cluster 
(http://hpc.nih.gov). This work was supported by the Intramural Research Programs of the NIH National Institute of Arthritis and Musculoskeletal and Skin Diseases and the National Human Genome Research Institute. We thank J. Fekecs for assistance rendering publication-ready figures.

\section{Author contributions}

M.K.H., M.Y.L., A.P.L., S.V. and J.A.S. conceived the study. M.K.H., M.Y.L., A.P.L., S.V., J.A.S., T.C., K.F., S.R.B., M. Pacilli, T.D., M.E.S., and H.H.K. participated in clinical study design. T.D., C.F., R.D.Y., C.D., Q.C., S.C., M. Stanley, P.B.B., S. Baskaran, the NISC Comparative Sequencing Program, R.M.W., D.J.S. and D.M.P. collected and/or processed samples. D.M.P., M.E.S., C.F., R.D.Y. and M. Park performed analyses. D.M.P., M.K.H., M.E.S., M.Y.L. and J.A.S. wrote the manuscript. All authors read, edited and approved the manuscript.

\section{Competing interests}

M.K.H. has been a co-investigator on several research studies for which Sage Products (now part of Stryker Corporation), Molnlycke and Medline provided chlorhexidine products at no charge to hospitals and skilled nursing facilities participating in the research. Neither M.K.H. nor her employer (Rush University Medical Center) received chlorhexidine products. M.Y.L. has received research support in the form of contributed product from Sage Products (now part of Stryker Corporation) and has received an investigator-initiated grant from the CareFusion Foundation (now part of BD). All other authors declare no competing interests.

\section{Additional information}

Extended data is available for this paper at https://doi.org/10.1038/s41591-021-01383-w. Supplementary information The online version contains supplementary material available at https://doi.org/10.1038/s41591-021-01383-w.

Correspondence and requests for materials should be addressed to J.A.S. or M.K.H.

Peer review information Nature Medicine thanks Tobias Hohl, Sudha Chaturvedi and Iliyan Iliev for their contribution to the peer review of this work. Alison Farrell was the primary editor on this article and managed its editorial process and peer review in collaboration with the rest of the editorial team.

Reprints and permissions information is available at www.nature.com/reprints. 


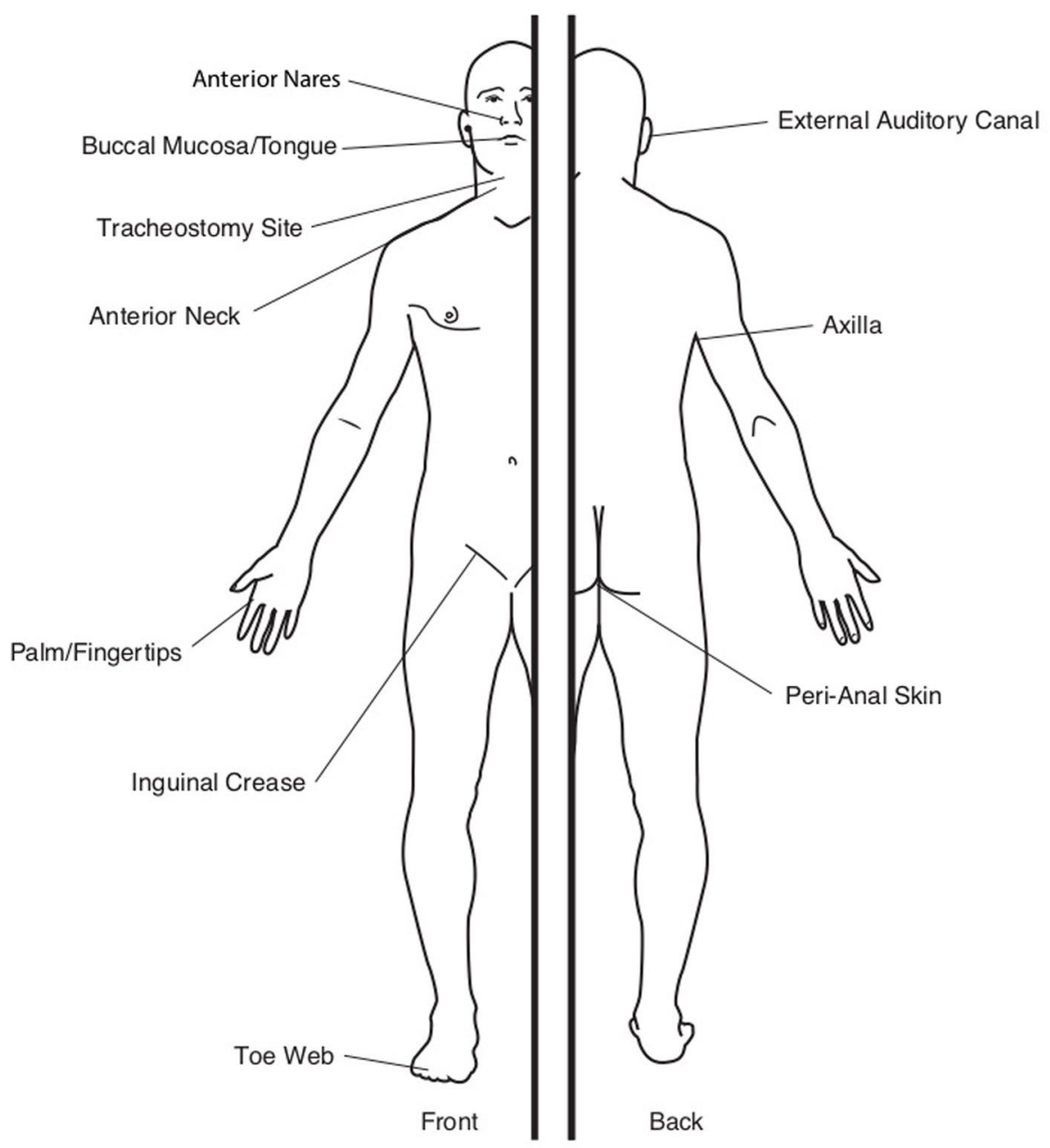

Extended Data Fig. 1 | Map of sample sites. We surveyed 10 body sites per subject, including the anterior nares (N), tracheostomy site (Tc), anterior neck $(\mathrm{Ne})$, palms/fingertips ( $\mathrm{Fg}$ ), buccal mucosa/tongue (Bu/To), inguinal crease (Ic), axilla ( $\mathrm{Ax}$ ), toe web (Tw), external auditory canal (Ea), and peri-anal $\operatorname{skin}(A n)$. 

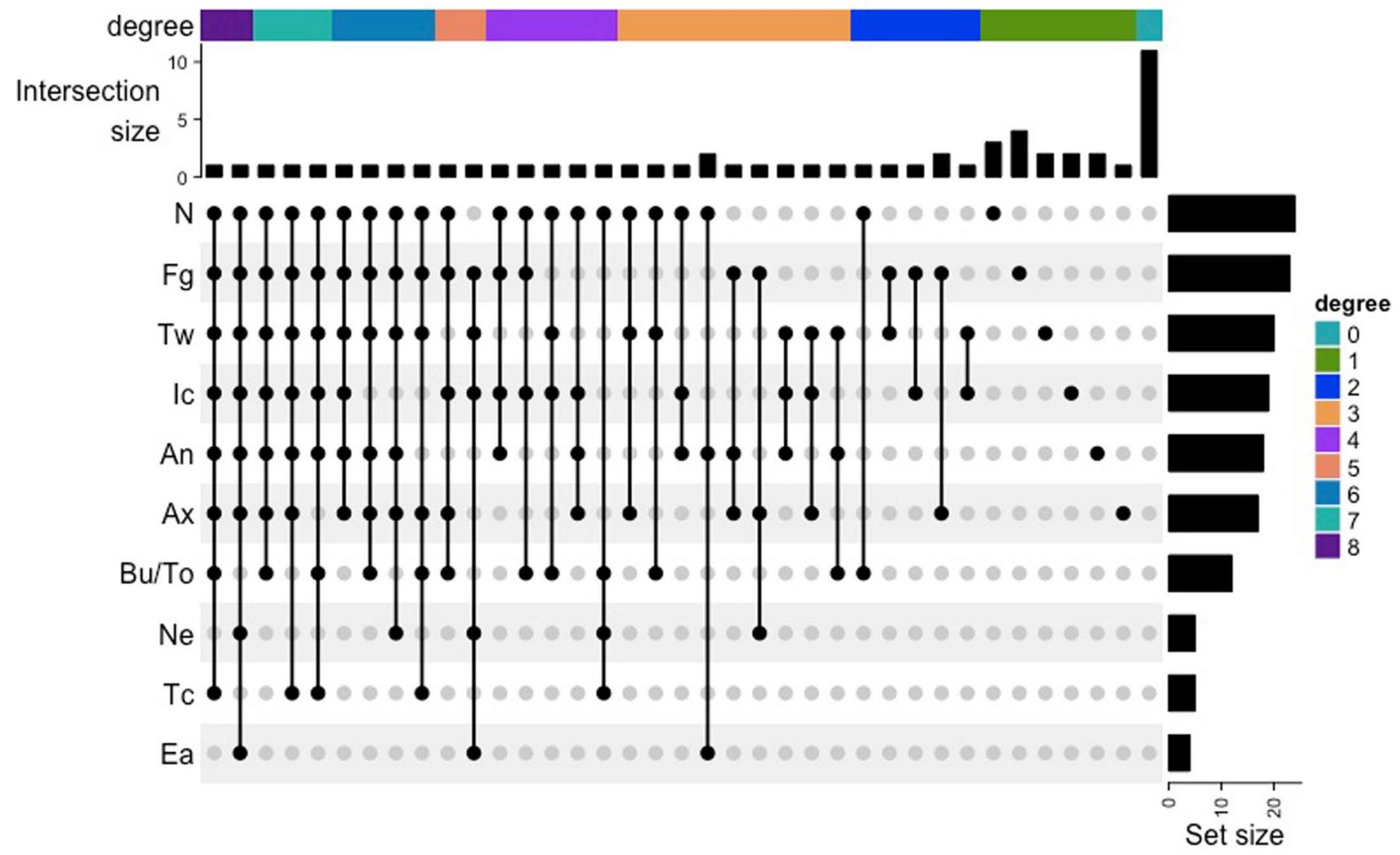

Extended Data Fig. 2 | Patterns of body site colonization visualized with UpSetR. Colors map to degree, a measure of the number of co-colonized sizes. A total of 36 distinct co-colonization patterns were observed, each arranged from the left to the right as a function of decreasing degree. The intersection size is the number of subjects whose body-site colonization matches the points connecting sites for each of the 36 unique co-colonization patterns. For example, the nares $(\mathrm{N})$ and fingertips/palm ( $\mathrm{Fg}$ ) are more frequently mono-colonized than any of the other sites while the buccal mucosa/tongue (Bu/To), neck $(\mathrm{Ne})$, tracheostomy site ( $\mathrm{Tc}$ ), and external auditory canal $(\mathrm{Ea})$ are never mono-colonized. Most patients have a distinct pattern of co-colonization with the most frequent pattern being singular colonization of the nares $(\mathrm{N})$ or fingertips/palm (Fg). The set size corresponds to the frequency of colonization for each site for the first time point. 


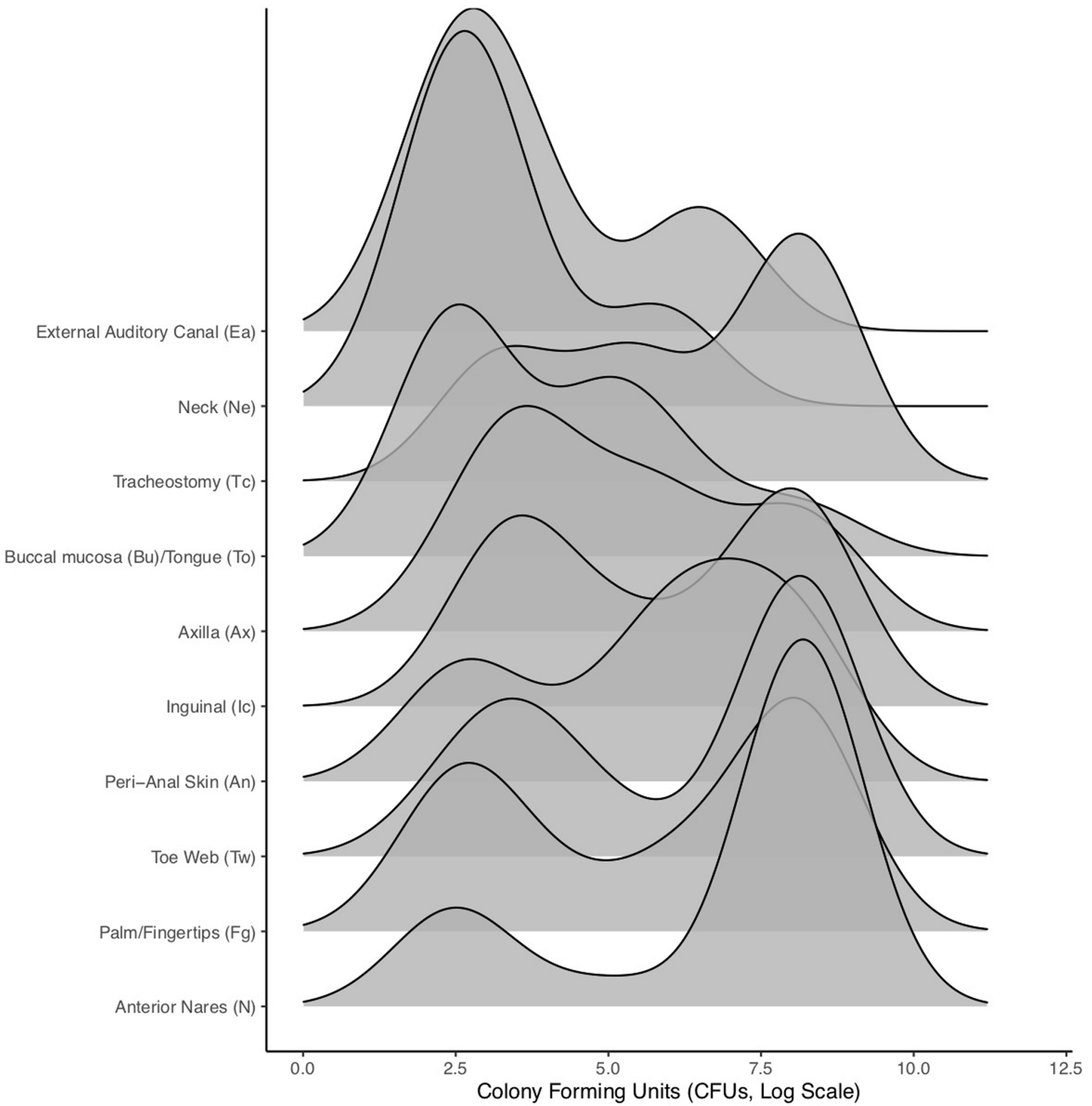

Extended Data Fig. 3 | Ridgeline plot of sample colony counts for each site during the first survey. The cumulative distribution for each ridgeline sums to 1, with peaks corresponding to peak bioburden (log colony forming units), for each site. For any given site, a bimodal distribution indicates a subset of subjects shared relatively high bioburdens and another a subset of subjects shared relatively low bioburdens. Sites with low level colonization include the external auditory canal and neck while sites having the highest bioburden include nares and inguinal crease. 


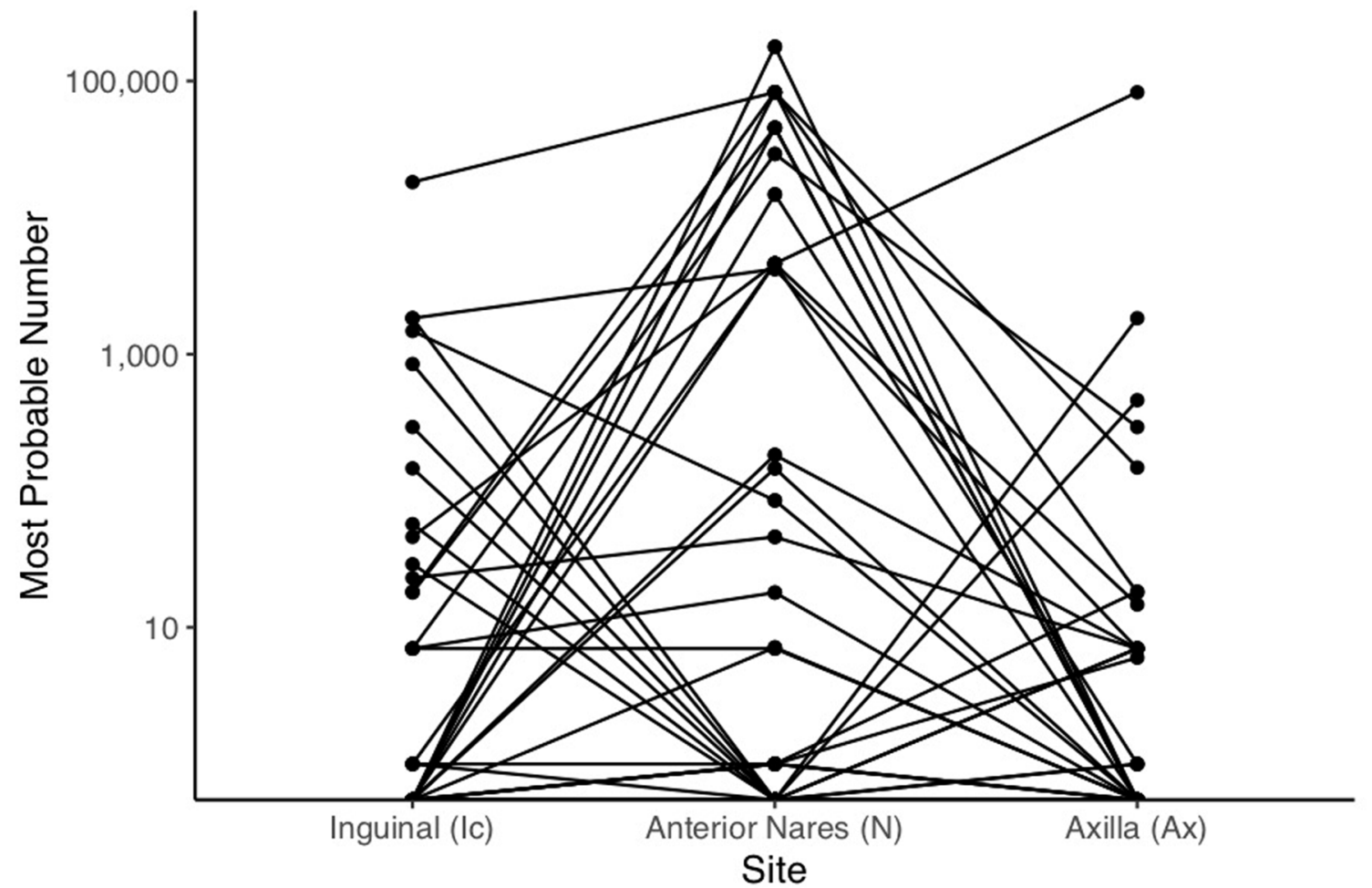

Extended Data Fig. 4 | Paired Most Probable Number (MPN) Analysis. MPN estimates are shown for the inguinal crease, anterior nares, and axilla. Data represented are from the first point prevalence survey. Each line represents an individual. Individual trajectories reveal a large number of individuals with high counts at the nares and either absent or low-level colonization at the axilla or inguinal crease. 


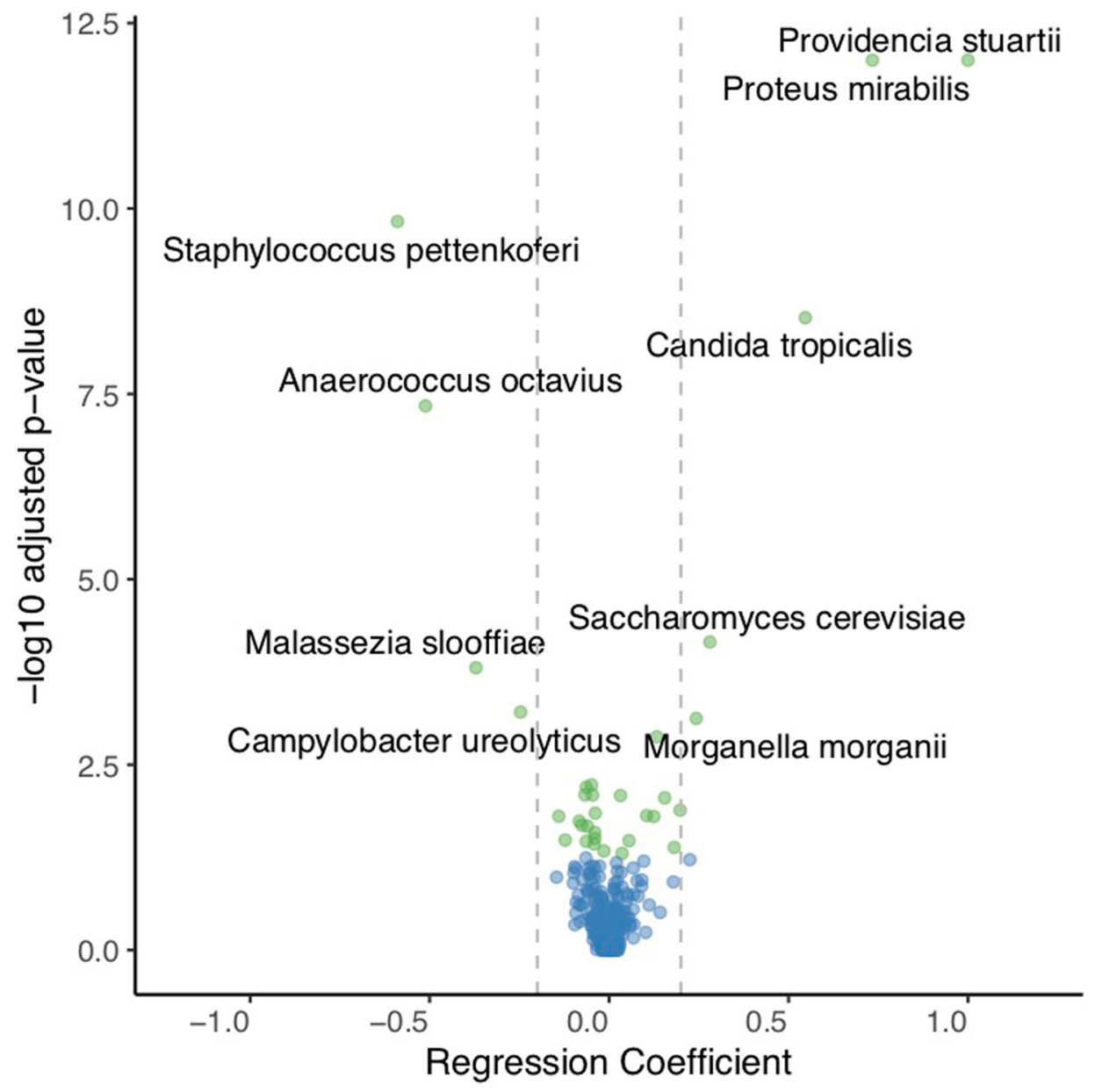

Extended Data Fig. 5 | Volcano plot of statistical significance (-Log adjusted p-value) against the regression coefficients from linear mixed effects models. Each point represents a regression coefficient for a bacterial or fungal species. The vertical lines demarcate regression coefficients of -0.2 and 0.2. Species having Holm adjusted p-values $<0.05$ are highlighted in green while non-significant taxa are in blue. Species exhibiting a positive association with CHG concentration (skin concentrations (estimate $>0.2$, Holm adjusted $p<0.05$ ) include Providencia stuartii, Proteus mirabilis, Candida tropicalis, Saccharomyces cerevisiae and Morganella morganii. Species exhibiting a negative correlation with CHG skin concentrations (estimate $<-0.2$, Holm adjusted p < 0.05) include Staphylococcus pettenkoferi, Anaerococcus octavius, Malassezia slooffiae, and Campylobacter ureolyticus. 
Wuf

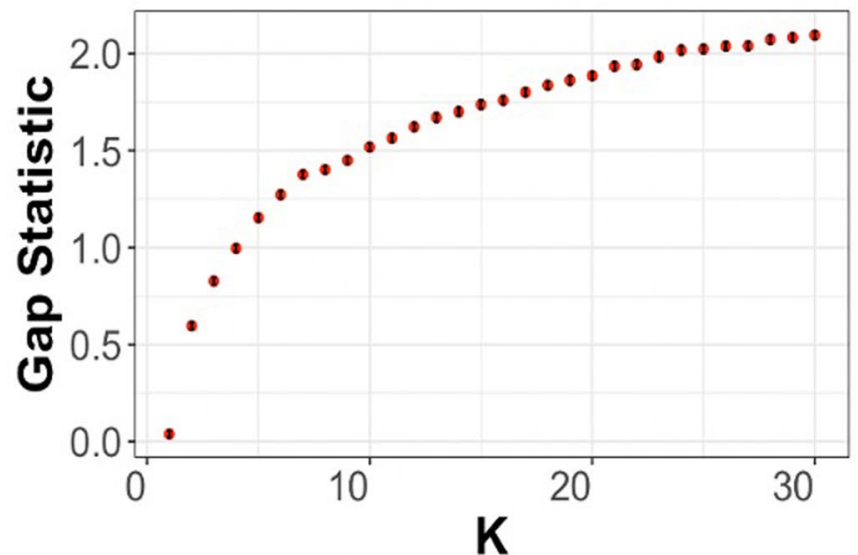

Bray

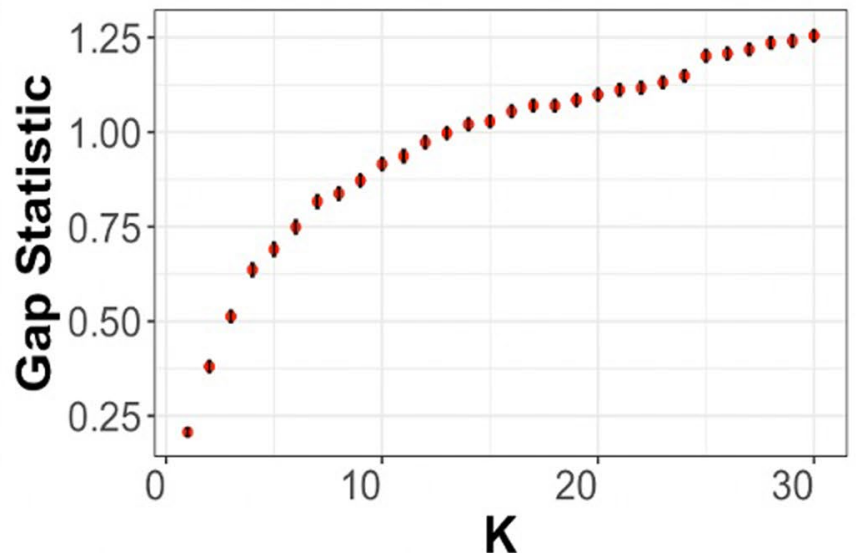

Extended Data Fig. 6 | Evaluation of the robustness of cluster formation. Weighted UniFrac (Wuf) and Bray-Curtis (Bray) distances were computed prior to partition around medoids analysis. The gap statistic ( $y$-axis) is plotted as a function of $\mathrm{K}$ ( $\mathrm{x}$-axis), defined as the number of clusters evaluated. The error bars correspond to the confidence interval generated on 1000 bootstraps. The optimal number of clusters corresponds to K where additional clusters fail to increase the gap statistic using both a phylogenetically aware distance metric (UniFrac) and Bray Curtis. 


\section{Fraction of Surveys Colonized}

$1 / 3$

$2 / 3$

$3 / 3$
Axilla

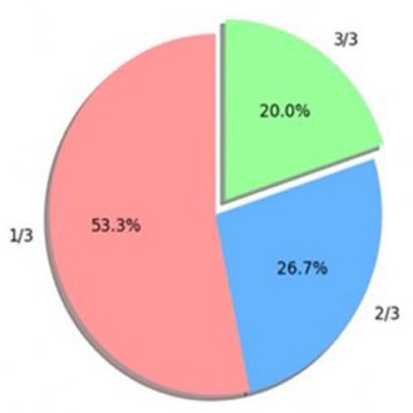

$\mathrm{N}=15$

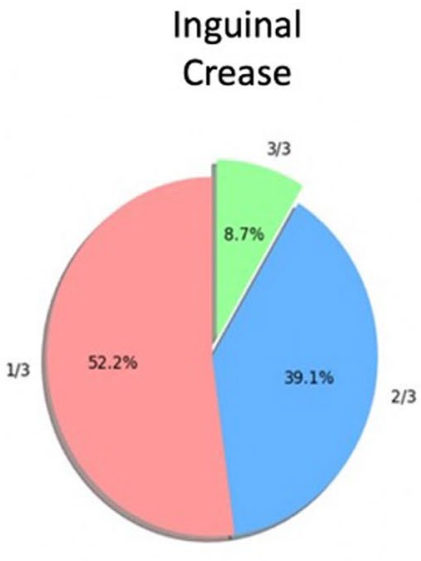

$N=23$
Anterior

Nares

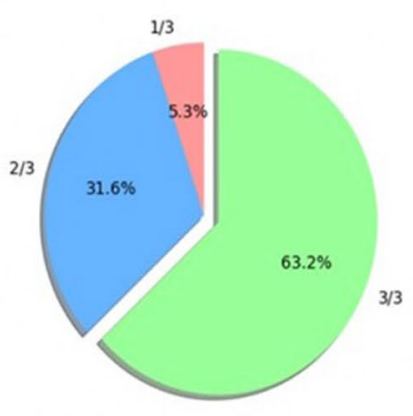

$\mathrm{N}=19$

Extended Data Fig. 7 | Temporal stability of $C$. auris colonization at the axilla, inguinal crease and anterior nares. Patients who were qualitatively categorized as positive at least once, based on MPN data, were categorized as positive in 1 of 3 (pink), 2 of 3 (blue) or 3 of 3 (green) surveys. The majority of subjects colonized at axilla and inguinal crease were positive in $1 / 3$ surveys while the majority of individuals colonized at the nares were positive at all 3 surveys. 


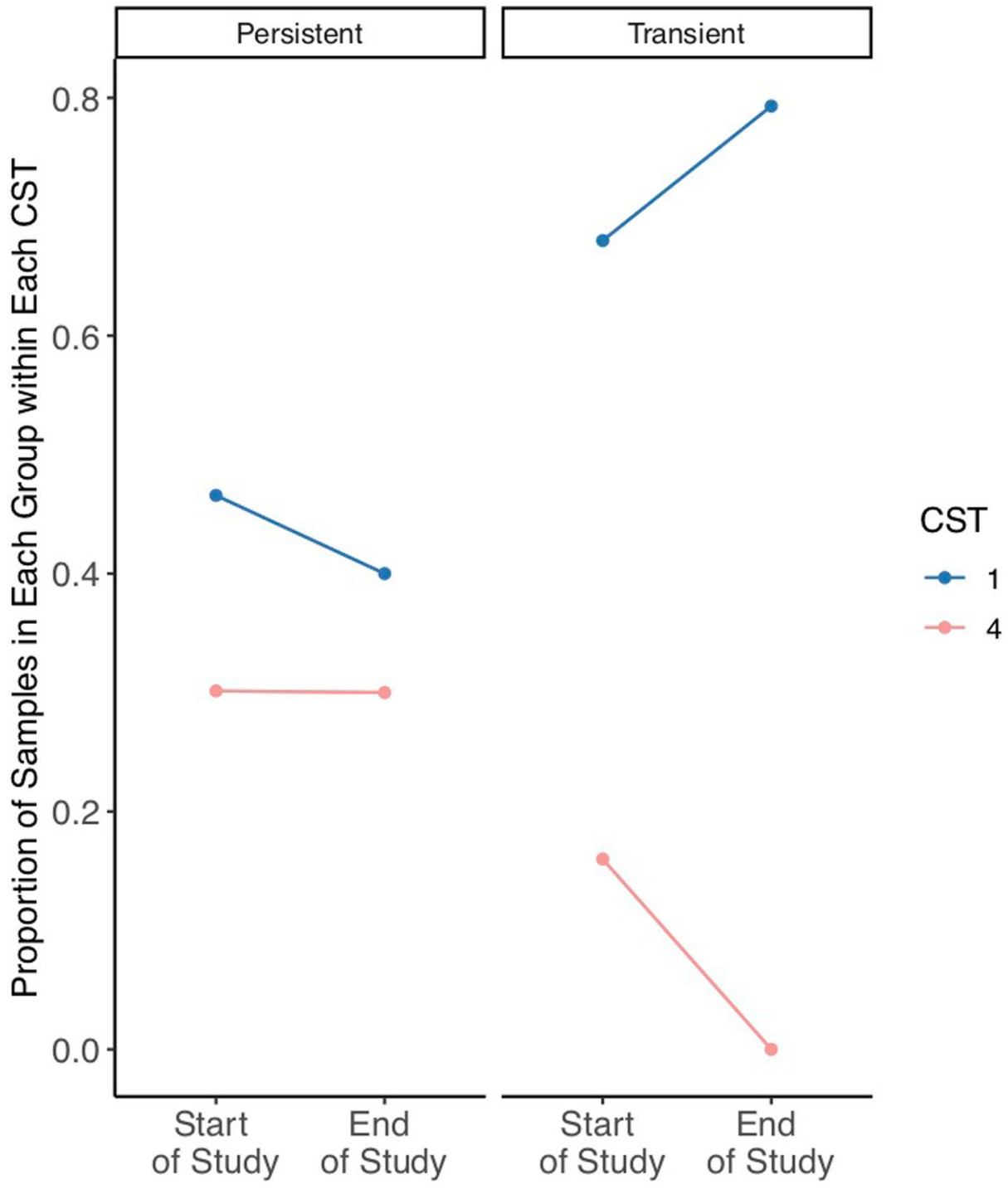

Extended Data Fig. 8 | Proportion of sites within each CST over time for individuals who were either transiently or persistently colonized. The proportion of samples dominated by C. auris (CST4) remained roughly constantly ( $30 \%$ ) for individuals who were persistently colonized (Left). In contrast, the proportion of sites dominated by $\mathrm{C}$. auris dropped from $16 \%$ to $0 \%$ from the first to the third time point in transiently colonized individuals (Right). Among those transiently colonized, the reduction in sites dominated by C. auris was accompanied by a concomitant increase in the proportion of sites dominated by commensal Malassezia species. Of special interest, the proportion of sites dominated by Malassezia species was higher across all time points for those who were transiently colonized compared to those persistently colonized. 


\section{Cauris relative Abundance}
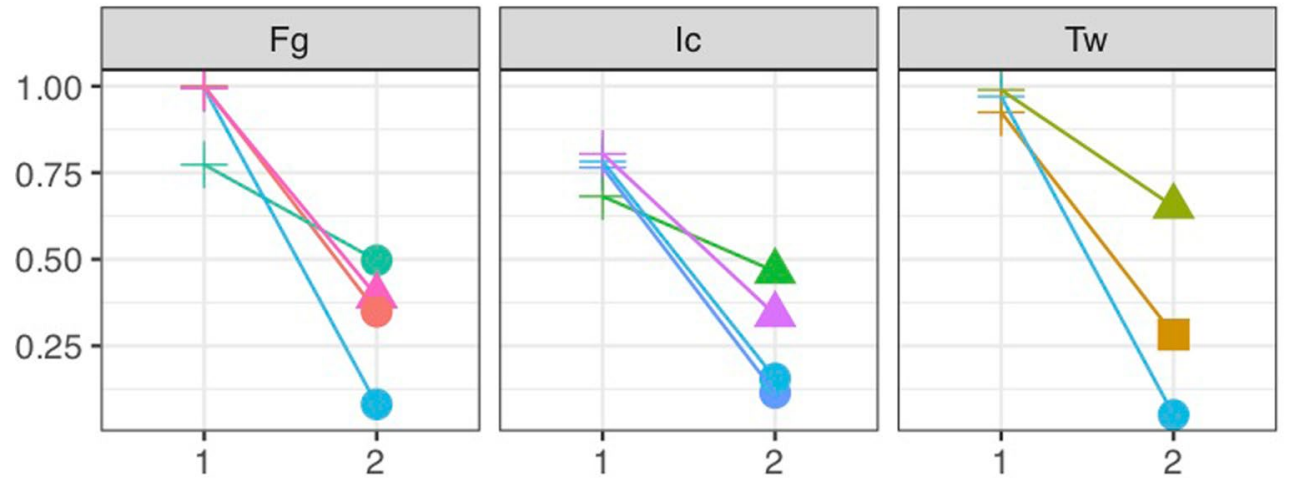

\section{Subject}

3

16

17

27

28

35

37

\section{Shannon Diversity}
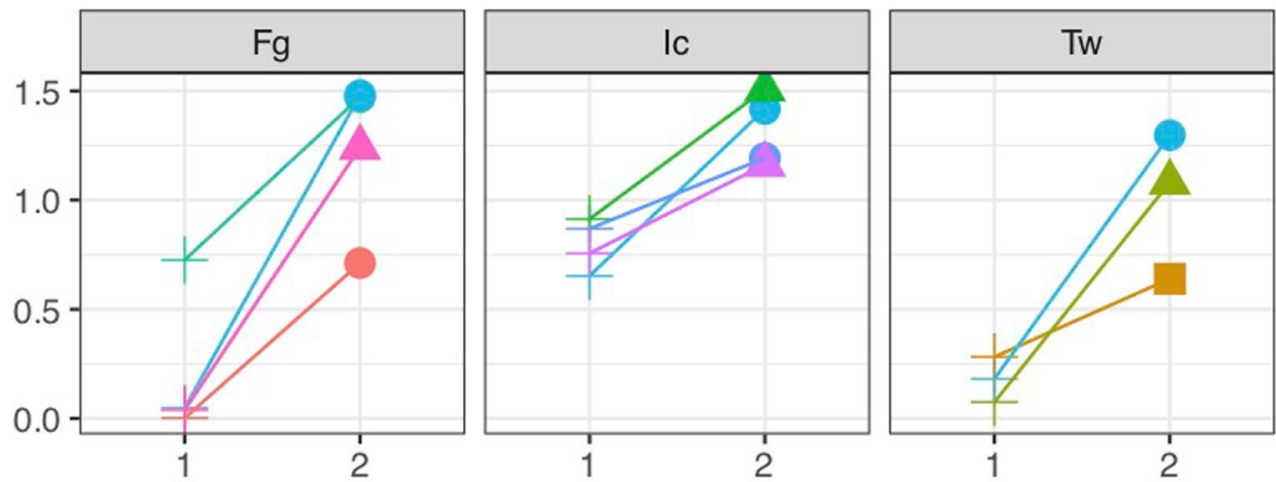

52

53

CST

- ${ }^{\text {CST1 }}$
- CST2 $^{\text {CST3 }}$
+ CST4

Extended Data Fig. 9 | Examination of the mycobiome at sites that transition away from C. auris domination between survey 1 and survey 2. Colors correspond to unique subjects. Shapes correspond to the mycobiome CST. Across all panels, survey 1 or 2 is shown on the $x$-axis. On the $y$-axes, the relative abundance of $C$. auris (top panel) or Shannon diversity (bottom panel) is depicted. For this analysis we looked exclusively at sites that transitioned away from domination by C. auris at the first survey (Survey 1) towards domination by another species at the second time point (Survey 2). 


\section{Reporting Summary}

Nature Research wishes to improve the reproducibility of the work that we publish. This form provides structure for consistency and transparency in reporting. For further information on Nature Research policies, see our Editorial Policies and the Editorial Policy Checklist.

\section{Statistics}

For all statistical analyses, confirm that the following items are present in the figure legend, table legend, main text, or Methods section.

$\mathrm{n} / \mathrm{a}$ Confirmed

$\bigotimes$ The exact sample size $(n)$ for each experimental group/condition, given as a discrete number and unit of measurement

\ A statement on whether measurements were taken from distinct samples or whether the same sample was measured repeatedly

The statistical test(s) used AND whether they are one- or two-sided

Only common tests should be described solely by name; describe more complex techniques in the Methods section.

\A description of all covariates tested

$\square$ \ A description of any assumptions or corrections, such as tests of normality and adjustment for multiple comparisons

$\square$ A full description of the statistical parameters including central tendency (e.g. means) or other basic estimates (e.g. regression coefficient)

AND variation (e.g. standard deviation) or associated estimates of uncertainty (e.g. confidence intervals)

$\square$ For null hypothesis testing, the test statistic (e.g. $F, t, r$ ) with confidence intervals, effect sizes, degrees of freedom and $P$ value noted

Give $P$ values as exact values whenever suitable.

Х $\square$ For Bayesian analysis, information on the choice of priors and Markov chain Monte Carlo settings

$\square$ \ For hierarchical and complex designs, identification of the appropriate level for tests and full reporting of outcomes

$\square \bigotimes$ Estimates of effect sizes (e.g. Cohen's $d$, Pearson's $r$ ), indicating how they were calculated

\section{Our web collection on statistics for biologists contains articles on many of the points above.}

\section{Software and code}

Policy information about availability of computer code

Data collection The clinical data were extracted from the facility's EMR- PointCareClick (PCC). Additional clinical data were recorded bedside on Case Report Forms prior to integration with extracted clinical data in REDCap 9.3.0.

Data analysis SAS v9.4 was used for clinical data analyses rendered in Table 1 and Figure 3b. Otherwise, all analyses were performed in R4.0.2. The amplicon sequencing data were pre-processed with cutadapt/2.10 prior to import into $R$ for all downstream analysis. $R$ packages included: BayesFactor_0.9.12-4.2, Biobase_2.48.0, BiocGenerics_0.34.0, broom.mixed_0.2.6, circlize_0.4.12, cluster version 2.1.2, coda_0.19-4, colorspace_2.0-0, ComplexHeatmap_2.4.3, compositions_2.0-1, cowplot_1.1.1, dabestr_0.3.0, dada2 version 1.13.0, DelayedArray_0.14.1, diagram version 1.6.5, DESeq2_1.28.1, dplyr_1.0.5, epiR_2.0.19, forcats_0.5.0, Formula_1.2-4, genefilter_1.70.0, GenomelnfoDb_1.24.2, GenomicRanges_1.40.0, ggalt_0.4.0, ggeffects_1.0.1, ggordiplots_0.3.0, ggplot2_3.3.3, ggpubr_0.4.0, ggrepel_0.9.1, ggridges_0.5.3, ggsci_2.9, gridExtra_2.3, gridGraphics_0.5-1, harrypotter_2.1.1, Hmisc_4.4-2, IRanges_2.22.2, jpeg_0.1-8.1, kableExtra_1.3.1, knitr_1.30, lattice_0.20-41, Ime4_1.1-26, ImerTest_3.1-3, magrittr_2.0.1, markovchain_0.8.5-4, MASS_7.3-53, Matrix_1.3-2, matrixStats_0.57.0, mixOmics_6.12.2, multcomp_1.4-15, mvtnorm_1.1-1, permute_0.9-5, phyloseq_1.32.0, purrr_0.3.4,

RColorBrewer_1.1-2, RDP Naive Bayesian Classifier 3, readr_1.4.0, reshape2_1.4.4, S4Vectors_0.26.1, scales_1.1.1, stringr_1.4.0,

SummarizedExperiment_1.18.2, survival_3.2-7, TH.data_1.0-10, tibble_3.1.0, tidyr_1.1.2, tidyverse_1.3.0, UpSetR_1.4.0, vegan_2.5-7, viridis_0.5.1 viridisLite_0.3.0, wesanderson_0.3.6, yarrr_0.1.5. The R code used to generate figures are provided as supplementary data (Supplementary Data Files 2-5) and can be found at https://github.com/dmap02/cauris_colonization. Package versions for all packages used in $\mathrm{R}$ are provided in the $\mathrm{html}$ rendering of Supplementary Data 2-5. Variant calling was performed using the following, as described in methods: MinKNOW 3.6.5, Guppy 3.6.0, seqtk version 1.2, canu-2.0, Racon v1.3.1, Medaka v1.0.3, Bowtie2 verion 2-2.4.2, Pilon v.1.23, Snippy, version 4.4.1, samtools version 1.11, freebayes 1.3 .5 , and bwa 0.7.17 
Policy information about availability of data

All manuscripts must include a data availability statement. This statement should provide the following information, where applicable:

- Accession codes, unique identifiers, or web links for publicly available datasets

- A list of figures that have associated raw data

- A description of any restrictions on data availability

The data supporting the results of this study are available in the NIH Short Read Archive, Bioproject accession number PRJNA672955: (https://

www.ncbi.nlm.nih.gov/bioproject/). The Refseq database v2.0 downloaded on May 2018 and the Unite sh_general_release_dynamic downloaded on 02-02-0219 were used to assign bacterial and fungal taxonomy, respectively, and can be found at https://github.com/dmap02/cauris_colonization. Intermediate data files including the OTU Table, taxonomy table, and sample data mapping file are included as Supplementary Data File 1.

\section{Field-specific reporting}

Please select the one below that is the best fit for your research. If you are not sure, read the appropriate sections before making your selection.

\Life sciences

Behavioural \& social sciences

Ecological, evolutionary \& environmental sciences

For a reference copy of the document with all sections, see nature.com/documents/nr-reporting-summary-flat.pdf

\section{Life sciences study design}

All studies must disclose on these points even when the disclosure is negative.

Sample size

Although this work was exploratory, we conducted an a priori power analysis to ensure that we would have sufficient power to detect notable differences in prevalence of $C$. auris colonization at different body sites, i.e. to test the null hypothesis of uniform distribution of $C$. auris across body sites. When we were planning the study, we anticipated participation of 35 patients with $C$. auris colonization and 25 patients without $C$. auris colonization during the 3 serial point prevalence surveys. We based these estimates on documented incidence, prevalence, and turnover rates at that time, and an assumption of $80 \%$ patient participation. Assuming participation of $35 \mathrm{C}$. auris colonized patients, true positive rates at the reference site ranging from $70 \%$ to $90 \%$, and $\alpha=0.05$, we calculated that we would have $80 \%$ power to detect relative risk in any comparison site ranging from .62 - .75 , i.e. $44 \%-68 \%$. In fact, we identified $45 \mathrm{C}$. auris positive and $12 \mathrm{C}$. auris negative patients during the first survey. Therefore, rather than seek new patients to test during each of the subsequent 2 surveys, we continued to follow the original 57 patient cohort, eventually identifying a total of $46 \mathrm{C}$. auris positive and $11 \mathrm{C}$. auris negative study participants.

Data exclusions All patients on the ventilator ward were eligible for study participation. No data for eligible subjects who completed the study were excluded.

Replication This was a single site, prospective, longitudinal observational study of an outbreak of Candida auris. A replication cohort was not enrolled and cannot be enrolled due to resource constraints associated with the COVID-19 pandemic.

Randomization This is a two cohort observational study with no randomization between cohorts. Individuals colonized by Candida auris were designated as part of the colonized cohort while individuals who were not colonized were recorded as such.

\begin{tabular}{l|l} 
Blinding & This is a two cohort observational study with no randomization between cohorts, so no blinding was necessary. All patients on the ventilator
\end{tabular} ward were eligible for study participation.

\section{Reporting for specific materials, systems and methods}

We require information from authors about some types of materials, experimental systems and methods used in many studies. Here, indicate whether each material, system or method listed is relevant to your study. If you are not sure if a list item applies to your research, read the appropriate section before selecting a response.

Materials \& experimental systems

Methods

$\mathrm{n} / \mathrm{a}$ Involved in the study

Х $\square$ Antibodies

Х $\square$ Eukaryotic cell lines $\mathrm{n} / \mathrm{a}$ Involved in the study

Х $\square$ ChIP-seq

\ $\square$ Palaeontology and archaeology

X $\square$ Flow cytometry

$\bigotimes \square$ Animals and other organisms

$\bigotimes \mid \square$ MRI-based neuroimaging

$\square \bigotimes$ Human research participants

\ $\square$ Clinical data

$\triangle \square$ Dual use research of concern 
Policy information about studies involving human research participants

Population characteristics

See Table 1 for population characteristics. The average age in years of the study cohort was 59 (SD \pm 14$)$. Of the 57 in the study cohort, $40.4 \%$ of participants were female and $70.2 \%$ were African American. We conducted 3 surveys, spaced at monthly intervals, between January 16 - April 8, 2019 on the 70-bed ventilator ward of a 300-bed ventilator-capable skilled nursing facility in Chicago, IL. Fifty-seven (72\%) of 79 unique residents on the ward during the study period participated, and of those participating, $49(86 \%)$ were found to be colonized with C. auris at one or more body site in one or more survey. Unadjusted bivariate associations of clinical factors associated with $\mathrm{C}$. auris colonization at the first survey are shown in Table 1. Overall, residents were older adults, had a history of colonization or infection with carbapenem-resistant bacteria (for which active surveillance on the ventilator was done periodically), and had long lengths of stay before the first date of sample collection. Subjects with $\mathrm{C}$. auris colonization were more likely to require an invasive medical device, such as gastrostomy tube, and $23(50 \%)$ C. auris colonized residents versus $0(0 \%)$ uncolonized residents were mechanically ventilated. Subjects with $\mathrm{C}$. auris colonization were more likely to have been exposed to antibacterial agents in the 90 days before first sample collection. No subject developed clinical infection with $C$. auris during the study period; 1 subject had a bloodstream infection recorded 6 weeks before the study started, and 2 subjects developed bloodstream infection with C. auris in the 6 months after the surveys concluded.

Recruitment

Participants were recruited from a ventilated ward of a skilled nursing facility (VSNF). All patients who were present in their rooms on the ventilator ward of the ventilator-capable skilled nursing facility at the time of the first survey were eligible for participation in the study. Eight patients declined participation in the study. There were no significant differences in average age or percentage women between those who declined participation and those who participated in the study. Investigation of residents in vSNFs may differ from those in acute care settings given the older age and chronic health conditions present in this vulnerable patient population.

Ethics oversight

This research complies with all relevant ethical human subjects research regulations. The study was reviewed and approved by the Rush University Institutional Review Board, which granted expedited review and waiver of informed consent. Study subjects did not receive monetary compensation for their participation.

Note that full information on the approval of the study protocol must also be provided in the manuscript. 